\title{
An MRI-powered and Controlled Actuator Technology for Tetherless Robotic Interventions
}

\author{
Panagiotis Vartholomeos, Christos Bergeles, Lei Qin, and Pierre E. Dupont
}

\begin{abstract}
This paper presents a novel actuation technology for robotically assisted MRI-guided interventional procedures. In the proposed approach, the MRI scanner is used to deliver power, estimate actuator state and perform closed-loop control. The actuators themselves are compact, inexpensive and wireless. Using needle driving as an example application, actuation principles and force production capabilities are examined. Actuator stability and performance are analyzed for the two cases of state estimation at the input versus the output of the actuator transmission. Closed-loop needle position control is achieved by interleaving imaging pulse sequences to estimate needle position (transmission output estimation) and propulsion pulse sequences to drive the actuator. A prototype needle driving robot is used to validate the proposed approach in a clinical MRI scanner.
\end{abstract}

Index Terms-MRI, magnetic actuation, medical robotics.

\section{INTRODUCTION}

$\mathbf{M}$ AGNETIC Resonance Imaging (MRI) produces high quality images without exposing the patient to ionizing radiation and also provides a single environment wherein surgical procedure planning, performance, and assessment can be conducted. These advantages are well recognized by the clinical and research communities, and have motivated research in robotically-assisted MR-guided interventions [Tsekos et al., 2007].

A variety of MR-compatible robotic systems have been designed. These systems are usually developed to treat a specific region of the body, such as the prostate, breast or brain, and most perform the task of needle insertion for either biopsies or interventional therapies [Masamune et al., 1995], [Tsekos et al., 2005], [Fischer et al., 2008], [Li et al., 2011], [Patriciu et al., 2007], [Song et al., 2011].

Conventional actuation principles involving electromagnetic motors are generally not MR-compatible since they both interfere with the magnetic fields used for imaging and also are unsafe since they experience high forces and torques when placed inside the scanner bore. Therefore alternative actuation principles are employed, such as ultrasonic motors, a technology which has been maturing over the last four decades [Barth,

P. Vartholomeos, C. Bergeles and P. E. Dupont are with the Department of Cardiovascular Surgery, Boston Children's Hospital, Harvard Medical School, Boston, Massachusetts, 02115, USA. \{firstname.lastname\}@childrens.harvard.edu. Lei Qin is with the Dana Farber Cancer Institute, Boston, Massachusetts, 02115, USA. 1qin2@partners.org. This work was supported by the the National Science Foundation under grant IIS-1208509, the Wyss Institute for Biologically Inspired Engineering and by the National Institutes of Health under grant R01HL073647. Portions of this work have been presented at IROS 2011 [Vartholomeos et al., 2011] and EMBC 2012 [Bergeles et al., 2012].
1973]. The first example of an MRI-safe ultrasonic-powered robot was the needle-insertion neurosurgical robot presented in [Masamune et al., 1995]. Since then, many successful MRIsafe robotic systems have been introduced utilizing ultrasonic motors [Chinzei et al., 2000], [Tsekos et al., 2007], [Tsekos et al., 2005], one of the most recent examples being the needlesteering of [Su et al., 2012], which employs non-harmonic piezoelectric actuators.

MR-compatibility measurements, however have demonstrated that ultrasonic motors can produce a large reduction in SNR when operated inside the MRI bore [Fischer et al., 2008]. This issue can be overcome by turning off motor power during imaging, thus complicating the implementation of realtime control, or by placing ultrasonic motors at a distance from the bore. In the latter case, however, flexibility, backlash and friction are introduced due to remote actuation of joints [Tsekos et al., 2005]. Recent results utilizing linear amplifiers and carefully shielded electronics suggest that simultaneous actuation and imaging is possible [Su et al., 2012] at the expense of increased system cost.

An alternative to ultrasonic powering is pneumatic actuation. Pneumatic actuators are MR-compatible and do not cause SNR reduction, but they do require a complicated installation that involves locating a control unit, power supplies, amplifiers and valves external to the MRI shielded room [Li et al., 2011], [Patriciu et al., 2007], [Tokuda et al., 2012]). Furthermore, the pneumatic transmission lines lower the bandwidth and, in combination with the spatial constraints of the MRI bore, complicate robot design [Song et al., 2011].

The contribution of this paper is to propose a new tetherless MR-compatible actuation technology in which the MRI scanner is programmed to deliver power, to estimate actuator state and to perform closed-loop control. The complete actuator system does not need any peripheral devices (amplifiers, drivers, pumps, etc.) inside or outside the MRI room. Control and sensing operations are accomplished using the MRI host computer, the MRI electronics and the gradient and RF coils.

Since the actuators leverage the existing MRI infrastructure, are completely self contained and can be fabricated from inexpensive components, they offer a simple and potentially disposable alternative to existing technologies. In addition, since the MR system provides both imaging and control of the interventional procedure using a common software interface, the integration of actuation and imaging is simplified.

Actuation is based on one or more small ferromagnetic bodies safely contained inside the motor that serve to convert the electromagnetic energy of the MR gradients into mechanical energy. The ferromagnetic bodies have a small volume and 
can be designed to be outside the imaging region of interest, thus not affecting imaging quality. MRI gradients have been previously employed for actuation of ferromagnetic particles in the vasculature [Martel et al., 2007], [Martel et al., 2009], [Arcese et al., 2011]. For example, closed-loop trajectory control of a $1.5 \mathrm{~mm}$ diameter ferromagnetic sphere in the carotid artery of a pig was demonstrated in [Martel et al., 2007], [Martel et al., 2009]. In these papers, the ferromagnetic particle is itself the robot.

Inspired by this work, the current paper proposes a means to use the same force production principles to design actuators that can power more general interventional robots. In the next section, the background theory on the generation of actuator forces by the MRI scanner is presented. Section III describes the principle of actuation and, using a 1 DOF needle driving robot as a motivational example, provides guidelines for relating clinically desired needle insertion forces to actuator and transmission design parameters.

Section IV compares the dynamic performance that can be achieved when estimating actuator state via motor rotor angle versus needle position. The former enables a nested controller structure in which an inner loop performs motor commutation (ensuring maximum output torque) while an outer loop performs needle position control. In contrast, while needle position estimation precludes commutator control (since the transmission amplifies needle position error), it is shown that open-loop commutation can be effective for the force profiles associated with needle tissue insertion. Consequently, this approach is employed in the remainder of the paper and a method for MRI-based needle position estimation is given in Section V. Section VI presents a prototype 1 DOF needle driving robot together with experiments validating the force capabilities of the actuator and demonstrating closedloop control based on needle position estimation. Conclusions appear in the final section of the paper.

\section{BACKGROUND THEORY}

When a ferromagnetic body is placed inside an MRI bore, it becomes magnetized due to the strong and uniform central field, $\vec{B}_{0}$, directed along the axis of symmetry of the bore. The magnetization across the volume of the body can be approximated as a lumped effect at the center of mass (CM) of the body [Abbott et al., 2007]. Consequently, the magnetized body is approximated by a magnetic dipole placed at its CM. For typical central field strengths, the magnetization magnitude asymptotically approaches the saturation magnetization value $\vec{M}_{s}$ of the material, and its direction points along the easy magnetization axis of the body, which depends on the shape anisotropy of the material. The magnetic torque and force acting on the body can be computed using the expressions for the torque $\vec{T}$ and force $\vec{F}$ acting on a magnetic dipole in an external field $\vec{B}=\vec{B}_{0}+\vec{B}_{g}$ :

$$
\begin{aligned}
\vec{T} & =V \vec{M}_{s} \times \vec{B} \\
\vec{F} & =V\left(\vec{M}_{s} \cdot \nabla\right) \vec{B}
\end{aligned}
$$

where $\vec{B}_{g}$ is the magnetic field generated by the gradient coils, $V$ is the magnetic volume of the material and $\vec{M}_{s}$ is

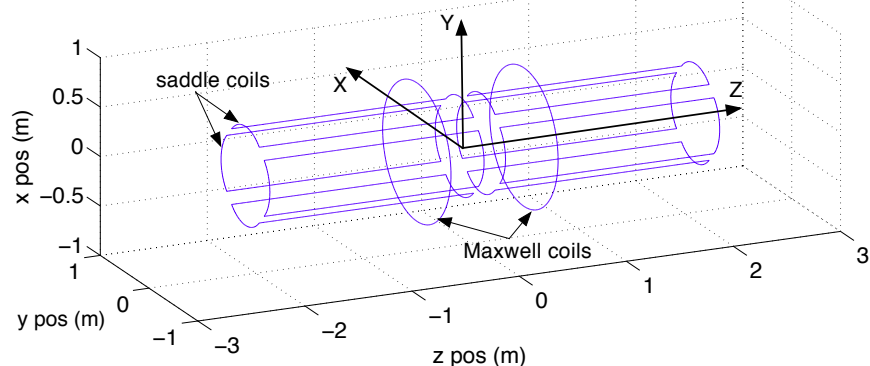

Fig. 1. MRI gradient coils configuration. The z-axis gradient are generated by a pair of Maxwell coils. The $\mathrm{x}$ and $\mathrm{y}$ gradients are generated by four pairs of saddle coils.

the saturated magnetization per unit volume of the material. The magnetic torque $\vec{T}$ tends to rotate the ferromagnetic body so that $\vec{M}_{s}$ aligns with the direction of the $\vec{B}_{0}$ field (the contribution of the $\vec{B}_{g}$ field to the torque $\vec{T}$ is negligible). Since the $\vec{B}_{0}$ field inside an MRI is fixed and cannot be controlled by the user, the torque $\vec{T}$ is not a valuable quantity for control purposes. The magnetic forces $\vec{F}$ depend on the spatial variation of the field $\vec{B}_{g}$, generated by the gradient coils. These comprise a pair of Maxwell coils and four pairs of saddle coils located orthogonal to each other as shown in Fig. 1.

Resolving the force $\vec{F}$ of (2) in the XYZ frame that is attached to the isocenter of the bore (see Fig. 1), yields:

$$
\left[\begin{array}{lll}
\vec{F}_{x} & \vec{F}_{y} & \vec{F}_{z}
\end{array}\right]=V M_{s z}\left[\frac{\partial B_{g x}}{\partial z} \frac{\partial B_{g y}}{\partial z} \frac{\partial B_{g z}}{\partial z}\right]
$$

where it has been reasonably assumed that $M_{s x}, M_{s y}<<$ $M_{s z}$. Equation (3) shows that the MRI forces acting on a ferromagnetic body are related to the three orthogonal gradients. The gradient coils are designed to generate (for imaging purposes) the three linearly independent gradients given by:

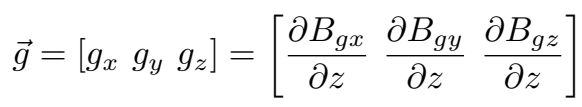

The Maxwell equations state that field lines must form closed loops, however, i.e. $\nabla \cdot \vec{B}=0$, and also that they must exhibit zero curl, i.e. $\nabla \times \vec{B}=0$, at any point outside the coil conductors. This implies that it is impossible to generate the gradients in (4) without also generating concomitant gradients. As a result, the complete field $\vec{B}_{g}$ is given by [Han et al., 2008]:

$$
\vec{B}_{g}(\vec{x})=(\vec{g} \cdot \vec{x}) \hat{z}+\mathbf{G}_{c} \vec{x}
$$

where $\vec{g}$ is as given in (4), $\vec{x}$ is the position vector from the origin if the XYZ frame, $\hat{z}$ is the unit vector along the $\mathrm{z}$-axis and $\mathbf{G}_{c}$ is a matrix containing the concomitant gradients and is given by:

$$
\mathbf{G}_{c}=\left[\begin{array}{ccc}
-g_{z} / 2 & 0 & g_{z} \\
0 & -g_{z} / 2 & g_{y} \\
0 & 0 & 0
\end{array}\right]
$$




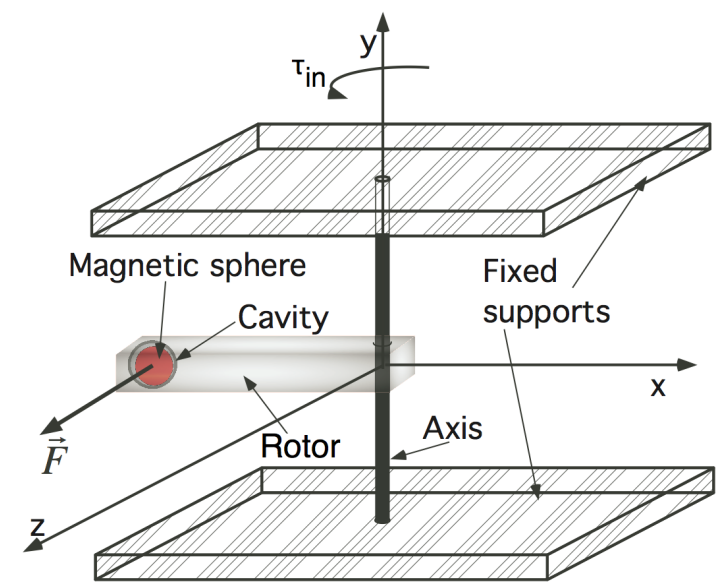

Fig. 2. Actuator schematic.

Differentiating the $x$ and $y$ components of Equation (5) with respect to the $z$ coordinate yields:

$$
\left[\frac{\partial B_{g x}}{\partial z}\right]=\left[\frac{\partial B_{g z}}{\partial x}\right],\left[\frac{\partial B_{g y}}{\partial z}\right]=\left[\frac{\partial B_{g z}}{\partial y}\right]
$$

Hence, the gradients of (3) are equal to the imaging gradients of (4) and, furthermore, all three are linearly independent. Thus they are capable of inducing 3 degree of freedom (DOF) motion in a ferromagnetic body.

\section{Actuator Design}

The proposed actuator is comparable to an electric motor. It consists of the stator, which, in this case, is comprised of the MRI scanner together with the stationary components of the actuator, and a rotor, which is the rotating portion of the actuator that contains the ferromagnetic material. As depicted in Fig 2, the rotor consists of a ferromagnetic object enclosed in a cavity in a lever arm that rotates at a fixed distance about an axis.

The object is contained in a cavity rather than embedded in the lever arm since, by (1), a large torque would be generated if rotation of the lever arm caused the magnetization vector of the sphere to rotate out of alignment with the $B_{0}$ field. Except in the case that the actuator axis was perfectly aligned with the central axis, such a torque would stall the actuator. Therefore, the ferromagnetic body must be free to rotate within its cavity. To minimize friction between the ferromagnetic body and cavity walls, the geometry of the ferromagnetic body should be selected as spherical and the surfaces of the sphere and cavity should be designed to minimize friction.

Rotation of the actuator is generated by applying magnetic field gradients to generate a force $\vec{F}$ on the ferromagnetic sphere as shown in Fig. 2. Assuming for simplicity that the $(x y z)$ frame of the actuator coincides with the $(X Y Z)$ frame of the isocenter of the MRI bore (Fig. 1), gravity is directed along the $y$-axis and so does not affect rotor motion. From the free-body diagram of Fig. 4, the single degree of freedom motion is described by the following scalar equation

$$
J \ddot{\theta}=-b \dot{\theta}-T_{f r}-T_{l}+r_{1} F \sin (\phi-\theta)
$$

Magnetic sphere is free to rotate within the cavity to align its magnetization to the external field $\mathrm{B}_{0}$

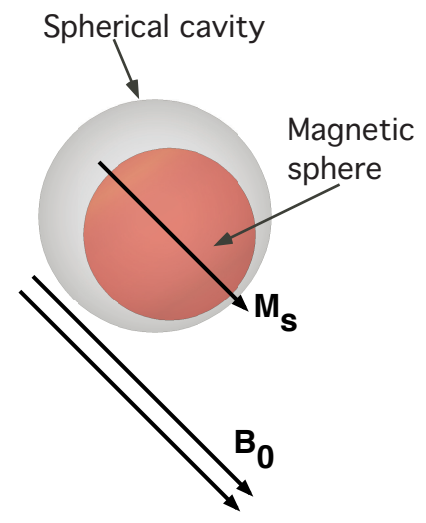

Fig. 3. Ferromagnetic sphere rolls and slides inside cavity to maintain alignment of its magnetic axis with $B_{0}$ field of scanner.

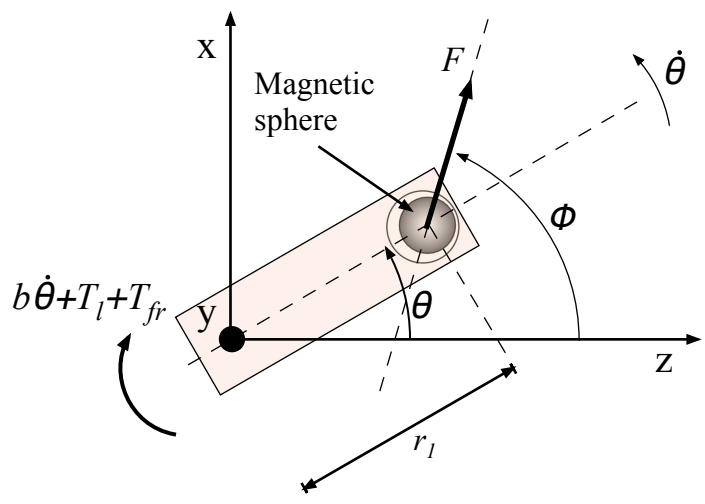

Fig. 4. Rotor coordinate frame and variables.

in which $J$ is the moment of inertia of the composite body that comprises the axis, the lever and the sphere, $r_{1}$ is as shown in Fig. 4, $b$ is the coefficient of viscous friction and $\theta$ is the angle of the rotor. The term $T_{f r}$ is the summation of all non-viscous friction terms as seen by the input including the friction component contributed by the ferromagnetic sphere when it slides inside the cavity. Also, $T_{l}$ is the load torque and and $\phi$ is the angle of the magnetic gradient. It is reasonably assumed that the eddy currents induced on the millimeter-scale chrome steel sphere are very small and can be neglected [Giffin et al., 2010].

The angle $\theta$ is called the mechanical angle and the angle $\phi$ is called the magnetic angle. Their difference is called the slip angle, $\psi$,

$$
\psi=\phi-\theta
$$

Note that actuator torque is maximized when $\psi=\pi / 2$. The values of $\vec{F}$ and $\phi$ are given by:

$$
\|\vec{F}\|=\sqrt{F_{x}^{2}+F_{y}^{2}+F_{z}^{2}}, \quad \phi=\tan ^{-1}\left(F_{x} / F_{z}\right)
$$

where $F_{x}, F_{y}$ are as given in (2).

\section{A. Needle Driving}

To examine the potential of this technology for roboticallyassisted MRI-guided procedures, it is considered here in the 


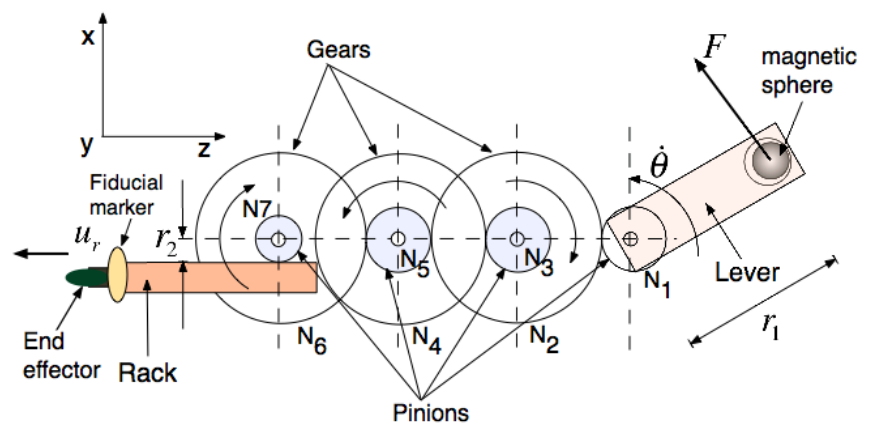

Fig. 5. Transmission unit incorporating gear train and rack and pinion elements.

context of needle insertion - specifically considering the forces needed to drive a needle into tissue. For this application, rotary actuator motion must be converted into linear needle motion using transmission elements. The type of transmission elements employed as well as the overall transmission ratio are design choices. As an illustrative example, Fig. 5 depicts one possible design, utilized later in the paper, employing a gear train together with a rack and pinion. To maximize output force, the $x-z$ plane of the rotor is selected to coincide with the $\mathrm{X}-\mathrm{Z}$ plane of the MRI bore. Otherwise, part of the magnetic force must compensate for gravity. If the output force has to be applied at an angle with respect to the $\mathrm{x}-\mathrm{y}$ plane, then bevel gears can be used to change the axis of rotation.

For the depicted transmission, the three gear ratios, $G_{1}, G_{2}, G_{3}$, are given by

$$
G_{1}=\frac{N_{2}}{N_{1}}, G_{2}=\frac{N_{4}}{N_{3}}, G_{3}=\frac{N_{6}}{N_{5}}
$$

in which $N_{i}, i=1, \ldots, 6$ are the numbers of gear teeth. The reduction ratio between $\dot{\theta}$ and the rack speed $u_{r}$ is then computed as

$$
R=\frac{u_{r}}{\dot{\theta}}=\frac{r_{2}}{G_{1} G_{2} G_{3}}
$$

in which $r_{2}$ is the radius of the $N_{7}$ pinion.

Independent of the type of transmission, the important design variables are its transmission ratio, $R$, and its efficiency, $\eta$. To design an actuator to produce a desired needle force, $F_{n}$, the design equation is given by

$$
F_{n} \leq \eta \frac{r_{1} F}{R}=\left.\eta \frac{r_{1}}{R} V M_{s z} \frac{\partial B_{g}}{\partial z}\right|_{\max }
$$

The force necessary to insert a needle varies with needle size and design as well as tissue type. Furthermore, the force can vary considerably during insertion with maxima occurring during puncture of elastic tissue layers. A number of papers have reported in vivo measurements of maximum needle forces. For example, [Washio and Chinzei, 2004] reported maximum forces of about $0.5 \mathrm{~N}$ inserting a $2 \mathrm{~mm}$ diameter needle into porcine spleen. [Maurin et al., 2004] reported maximum forces for manual $18 \mathrm{G}$ needle insertion in pigs of $0.7 \mathrm{~N}$ for liver, $0.74 \mathrm{~N}$ for kidney and $0.83 \mathrm{~N}$ for pancreas. Percutaneous insertion forces are higher; the same authors reported maximum forces for percutaneous liver insertion to be $3.73 \mathrm{~N}$ when performed manually and $1.89 \mathrm{~N}$ when performed
TABLE I

Design SCENARIO PARAMETERS FOR Producing Clinically RELEVANT FORCES.

\begin{tabular}{llr} 
& Parameter & Value \\
\hline \hline Common & Saturated magnetization, $M_{s}\left[A m^{-1}\right]$ & $1.36 \cdot 10^{6}$ \\
Parameters & Maximum MRI gradient $[\mathrm{mT} / \mathrm{m}]$ & 40 \\
& Gear ratio, $G_{1} G_{2} G_{3}$ & 125 \\
& Rotor radius, $r_{1}[\mathrm{~mm}]$ & 18 \\
\hline \hline Design 1 & Desired force, $F_{n}[\mathbf{N}]$ & $\mathbf{0 . 5}$ \\
\hline & Efficiency $[\eta]$ & 0.2 \\
& Pinion radius, $r_{2}[\mathrm{~mm}]$ & 5 \\
& Ferromagnetic sphere radius $[\mathrm{mm}]$ & 3 \\
\hline \hline Design 2 & Desired force, $F_{n}[\mathbf{N}]$ & $\mathbf{2 0}$ \\
\hline & Efficiency $[\eta]$ & 0.5 \\
& Pinion radius, $r_{2}[\mathrm{~mm}]$ & 10 \\
& Ferromagnetic sphere radius $[\mathrm{mm}]$ & 6
\end{tabular}

robotically. Maximum forces for in vivo human prostate capsule puncture during brachytherapy have been reported to be about $8.9 \mathrm{~N}$ using $18 \mathrm{G}$ needles and $15.6 \mathrm{~N}$ for $17 \mathrm{G}$ needles [Podder et al., 2006].

Equation (13) provides the means to determine whether or not the proposed technology can produce clinically relevant forces. Table I presents two potential designs computed using (13) that span the range of reported maximum force data. These designs assume the standard maximum clinical MRI gradient of $40 \mathrm{mT} / \mathrm{m}$ and utilize a single chrome steel sphere. For simplicity, both designs employ a gear ratio, $G_{1} G_{2} G_{3}=125$ and a rotor radius of $r_{2}=18 \mathrm{~mm}$. Design 1 , corresponding to the prototype presented in Section VI, is intended to produce maximum forces of $0.55 \mathrm{~N}(>0.5 \mathrm{~N})$, sufficient for porcine spleen insertion. Design 2 specifies a maximum force of $20 \mathrm{~N}$, which would be sufficient for human prostate capsule puncture.

The factor of 40 difference in output force between these designs is achieved by varying three of the design parameters. First, the transmission efficiency is increased by a factor of 2.5 from the low initial value of 0.2 that is characteristic of low-precision components to the very conservative value of 0.5 which can be easily exceeded using high-precision transmission elements. Doubling the pinion radius to $1 \mathrm{~cm}$ keeps the overall transmission size small while doubling the transmission ratio. Finally, doubling the ball radius to $6 \mathrm{~mm}$ provides a factor of 8 increase in volume and force.

While both designs utilized the current maximum gradient value of clinical scanners, $40 \mathrm{mT} / \mathrm{m}$, it is interesting to note that higher gradients may become available in the pursuit of increased imaging resolution. For example, in vivo brain measurements using $300 \mathrm{mT} / \mathrm{m}$ gradients produced by $55 \mathrm{~cm}$ diameter gradient coils have recently been demonstrated [McNab et al., 2012]. Such systems would enable an order of magnitude increase in actuator forces or, alternately, a reduction in transmission ratio and size.

\section{B. MRI Compatibility}

Since the actuator technology utilizes magnetic gradients to produce propulsive forces on ferromagnetic material located within the actuator, it is necessary to ensure that the resulting actuators are MRI compatible. MRI compatibility typically 
involves satisfying three types of constraints: (1) maintaining image quality, (2) ensuring safety and (3) meeting geometric constraints. In the proposed approach, there is an additional constraint: (4) imaging pulse sequences should not produce unintentional actuator motions or forces.

The first constraint implies that insertion and, ideally, operation of the actuator inside the scanner should not decrease image signal to noise ratio (SNR) in the imaging region of interest. This constraint is met in the proposed approach by placing the ferromagnetic material at a sufficient distance from the imaging region and by proper selection of actuator structural components, e.g., plastics.

To ensure the safety of the patient, medical personnel and the scanner, any ferromagnetic material used in an actuator should be constrained so as avoid any risk of creating an uncontrolled motion of the ferrous material itself or of the actuator, e.g., projectile motion. This is accomplished by sealing the ferrous material in a cavity so that it cannot escape the actuator and by properly fixing the actuator with respect to the scanner and the patient.

The geometric constraints of MRI compatibility relate to ensuring that the size of the actuator / robot does not create physical interference with either the patient or the scanner. While the proposed actuators do require mechanical transmissions to amplify output forces and torques, overall size remains modest. For example, the prototype needle driving robot presented later in the paper is sufficiently small to be placed inside the scanner bore at any desired location on a patient's body.

Employing electromagnetic actuation introduces a new compatibility requirement in that the imaging sequences employed during actuation should not cause unintended actuator motions or forces. This can be achieved by developing imaging pulse sequences using gradient pulse magnitudes and/or directions that are non-propulsive. Alternately, the pulse sequence may be balanced such that it has no net propulsive effect. These techniques are utilized in the actuator state estimation of section $\mathrm{V}$.

\section{Dynamic Behavior for Open- AND Closed-Loop CONTROL of Slip ANGLE}

The position control loop for needle driving can be closed using MRI-based sensing of actuator position. Position sensing can either be achieved at the transmission output (needle position) or at the input of the transmission (rotor angle). Directly sensing needle position is desirable since it is the clinical quantity of interest and avoids any risk of insertion errors arising from image-based miscounting of rotor revolutions. MRI image-based tracking is also simpler than rotor tracking owing to its linear motion and lower velocity.

A disadvantage of needle position sensing, however, is that it does not provide an accurate estimate of rotor angle owing to the transmission ratio. Estimation of rotor angle can be important since production of maximum actuator torque depends on directing the magnetic gradient perpendicular to the rotor, i.e., regulating slip angle to $\psi=\pi / 2$. In electric motors, this is referred to as commutation and is accomplished either mechanically (brushed motors) or electronically by closing a feedback loop around rotor angle. Thus, needle position control theoretically requires both an inner commutation loop to control slip angle and an outer loop to control needle position.

While MRI-based commutation control is both feasible and desirable in some circumstances [Bergeles et al., 2013], openloop slip angle control can be sufficient in some applications. To understand the differences, this section compares closedloop and open-loop control of slip angle. It is shown that open-loop slip angle control can be stable for needle loading scenarios similar to those experienced during tissue insertion. Furthermore, the magnitude of applied needle forces can approach that of closed-loop control. For these analyses, (8) is rewritten in terms of needle force, $F_{n}$ as

$$
\begin{aligned}
& J \ddot{\theta}=-b \dot{\theta}-T_{f r}-R F_{n}+r_{1} F \sin (\phi-\theta) \\
& u_{r}=R \dot{\theta}
\end{aligned}
$$

Here, $R$ and $u_{r}$ are defined in (12). The terms $J$ and $b$ are the summation of the inertia and viscous friction terms, respectively, of all transmission stages as seen by the input of the system. All other terms are as defined above.

\section{A. Closed-loop control of slip angle (commutation control)}

During commutation control, slip angle is regulated to $\psi=$ $\pi / 2$. In this case, the steady-state output linear velocity $u_{r}$ is given by:

$$
\begin{array}{r}
u_{r}=-\frac{R^{2}}{b} F_{n}+R\left(\frac{r_{1} F}{b}-\frac{T_{f r}}{b}\right) \\
=-\frac{R^{2}}{b} F_{n}+R\left(\frac{r_{1} V M_{s} B_{\text {grad }}}{b}-\frac{T_{f r}}{b}\right)
\end{array}
$$

where

$$
B_{\text {grad }}=\sqrt{\left(\frac{\partial B_{g z}}{\partial z}\right)^{2}+\left(\frac{\partial B_{g x}}{\partial z}\right)^{2}}
$$

Equation (16) reveals that under commutation control, the output velocity $u_{r}$ is linearly related to the output force $F_{n}$ through a negative proportionality constant. The maximum permissible output load (stall or blocking force) is obtained for $u_{r}=0 \mathrm{~ms}^{-1}$ and is given by:

$$
F_{n}^{\max }=\left(r_{1} F-T_{f r}\right) / R
$$

Setting the output force $F_{n}$ to zero yields the maximum output velocity $u_{r}^{\max }$. This maximum output velocity corresponds to a maximum rotational velocity of the lever $\dot{\theta}^{\max }=$ $u_{r}^{\max } / R$, which in turn gives the maximum gradient frequency $\omega^{\max }$ for a given $B_{\text {grad }}$ :

$$
\omega^{\max }=\left(r_{1} F-T_{f r}\right) / b
$$




\section{B. Open-loop control of slip angle}

The preceding subsection requires a nested control structure in which an inner control loop regulates slip angle while an outer loop controls actuator position or velocity. This subsection addresses the simpler case (from an implementation perspective) in which slip angle is not controlled. To understand if this approach may suffice for some interventional applications, this subsection assesses stability for two important cases. The first is when a constant load is applied to an actuator that is driven at constant velocity. The second case corresponds to a linear elastic load driven at constant velocity. This latter case is comparable to the loading experienced by a needle pressing against tissue prior to puncture. In both cases the MRI scanner is programmed to generate gradients rotating in the $\mathrm{X}-\mathrm{Z}$ plane at constant frequency $\omega$. The gradients are given by:

$$
\left[\frac{\partial B_{g x}}{\partial z} \frac{\partial B_{g z}}{\partial z}\right]=B_{\text {grad }}[\cos \omega t \quad \sin \omega t]
$$

1) Constant-velocity Constant-load Stability: In the open loop case, stable operation of the actuator implies the rotor synchronizes with the rotating gradient frequency, $\omega$. To analyze the steady-state behavior of the system in response to the gradients of (20), the system (14) is rewritten in terms of the slip angle, $\psi=\phi-\theta$, in which $\phi=\omega t$. Time is also nondimensionalized to reduce the number of free parameters using the non-dimensionalized time $\tau$.

$$
\tau=\sqrt{\frac{r_{1} F}{J}} t
$$

The resulting equation is given by

$$
\ddot{\psi}+\alpha \dot{\psi}+\sin \psi=\beta
$$

where $\dot{\psi}$ and $\ddot{\psi}$ are now differentiated with respect to $\tau$ and

$$
\begin{aligned}
\alpha & =\frac{b}{\sqrt{r_{1} F J}} \\
\beta & =\left(R F_{n}+b \omega+T_{f r}\right) / r_{1} F
\end{aligned}
$$

Insight into the behavior of the rotor can be gained by observing that (22) is the equation of a damped pendulum experiencing a constant applied torque and that slip angle $\psi$ corresponds to the angle of the pendulum. Thus, it can be anticipated that the system will possess one stable and one unstable equilibrium point at $\psi=\{0, \pi\}$, respectively, when the applied torque $\beta=0$. Referring back to Fig. 4, these configurations correspond to the rotor being aligned and anti-aligned, respectively, with the direction of the rotating magnetic force, $F$. For a non-zero applied torque $\beta$, these equilibria will shift such that the torque generated on the rotor by $F$ balances $\beta$. This reasoning is demonstrated in the analysis below.

a) Determination of equilibrium points: Expressing (22) in state-space form with $x_{1}=\psi$ and $x_{2}=\dot{\psi}$ yields

$$
\begin{aligned}
& \dot{x_{1}}=x_{2} \\
& \dot{x_{2}}=-\alpha x_{2}-\sin x_{1}+\beta
\end{aligned}
$$

The equilibrium points of the states $\left(x_{1}, x_{2}\right)$ are $\left(\psi^{*}, 0\right)$ and satisfy

$$
\dot{x}_{1}=\dot{x}_{2}=0
$$

and, as expected, are given by:

$$
\psi^{*}=\sin ^{-1} \beta
$$

The condition, $|\beta| \leq 1$, for existence of a solution can be seen from (24) to require that the total torque arising from friction and the applied load not exceed the maximum magnetic torque produced by the magnetic force $F$ acting over the rotor moment arm radius of $r_{1}$.

Equation (27) yields two isolated equilibrium points $\left\{\psi_{1}^{*}, \psi_{2}^{*}\right\}$, in the range $[-\pi, \pi]$, as given below.

$$
\begin{array}{ll}
\psi_{1}^{*}=\sin ^{-1} \beta, & \left|\psi_{1}^{*}\right| \leq \pi / 2 \\
\psi_{2}^{*}=\pi-\psi_{1}^{*}, & \left|\psi_{2}^{*}\right| \geq \pi / 2
\end{array}
$$

b) Stability analysis of the equilibrium points: Linearizing (26) about the equilibrium points yields the error equation

$$
\dot{e}=A e, \quad e=\left[\psi-\psi^{*}, \dot{\psi}-0\right]^{T}
$$

where the stability matrix $A$ is given by

$$
A=\left[\begin{array}{cc}
0 & 1 \\
-\cos \psi^{*} & -\alpha
\end{array}\right]
$$

The eigenvalues $\lambda_{1,2}$ of matrix $A$ are given by

$$
\lambda_{1,2}=\frac{-\alpha \pm \sqrt{\alpha^{2}-4 \cos \psi^{*}}}{2}
$$

Thus, an equilibrium value $\psi^{*}$ is locally asymptotically stable for $\left|\psi^{*}\right|<\pi / 2$, locally marginally stable for $\psi^{*}=\pi / 2$ and locally unstable for $\left|\psi^{*}\right|>\pi / 2$. Hence, for the realistic case of $\alpha>0$, the equilibrium $\psi_{1}^{*}$ in (28) is asymptotically stable and $\psi_{2}^{*}$ in (29) is unstable.

Fig. 6 illustrates the pendulum analogy in the context of the actual system. The magnetic gradient, rotating with angular velocity $\omega$, is depicted at the instant in which it is directed along the $z$ axis such that the magnetic angle $\phi=0$ and the rotor angle $\theta=-\psi$. In this snapshot view, the magnetic gradient is analogous to gravity acting on a pendulum which also experiences an opposing constant load torque of magnitude $r_{1} F \beta$. The magnetic and load torques balance at the rotor configurations $\psi_{1}^{*}$ and $\psi_{2}^{*}$, which are arranged symmetrically with respect to the $x$ axis. As the load torque increases, the equilibria move toward the negative $x$ axis where they coalesce at $\psi_{1}^{*}=\psi_{2}^{*}=\pi / 2$, the configuration at which magnetic torque is a maximum. This also corresponds to the configuration at which $\psi_{1}^{*}$ becomes unstable. Thus, stability can only be maintained for load torques that are less than the maximum magnetic torque.

c) Domain of attraction of equilibrium points: It is important to understand how far the region of stability extends around the stable equilibrium point $\psi_{1}^{*}$. For example, such an analysis will reveal if stable rotation will result if the rotor is started from rest or a perturbation in applied force drives the system from the equilibrium point. Since (25) is second order and autonomous, the domain of attraction can be geometrically represented in the phase plane. Fig. 7 illustrates the stable and unstable regions for three sets of $(\alpha, \beta)$ values, namely: $\left\{\left(\alpha_{1}, \beta_{1}\right)=(0.96,0)\right\},\left\{\left(\alpha_{2}, \beta_{2}\right)=\right.$ $(91.1,0.0096)\},\left\{\left(\alpha_{3}, \beta_{3}\right)=(169.2,0.018)\right\}$. For each value, 


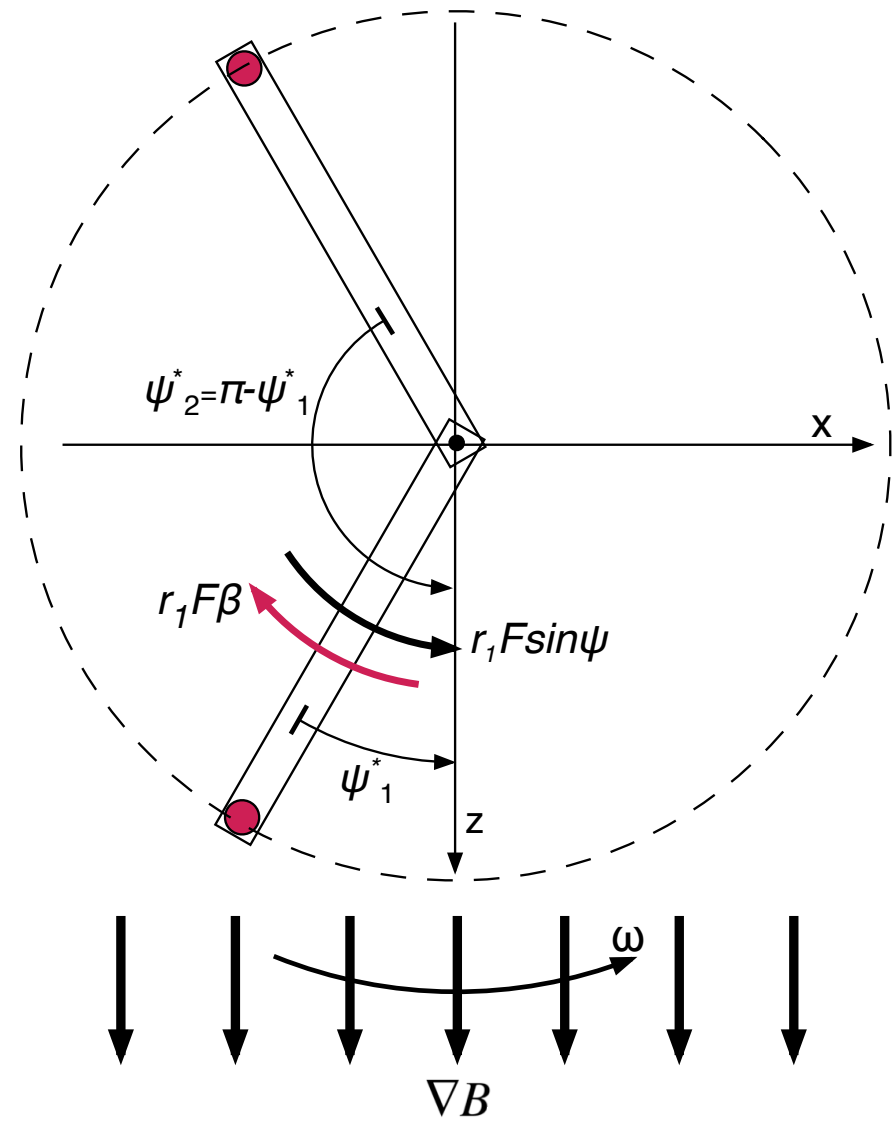

Fig. 6. Rotor equilibrium configurations for a rotating magnetic gradient depicted at the instant when magnetic angle $\phi=0$ so that rotor angle $\theta=$ $-\psi$.

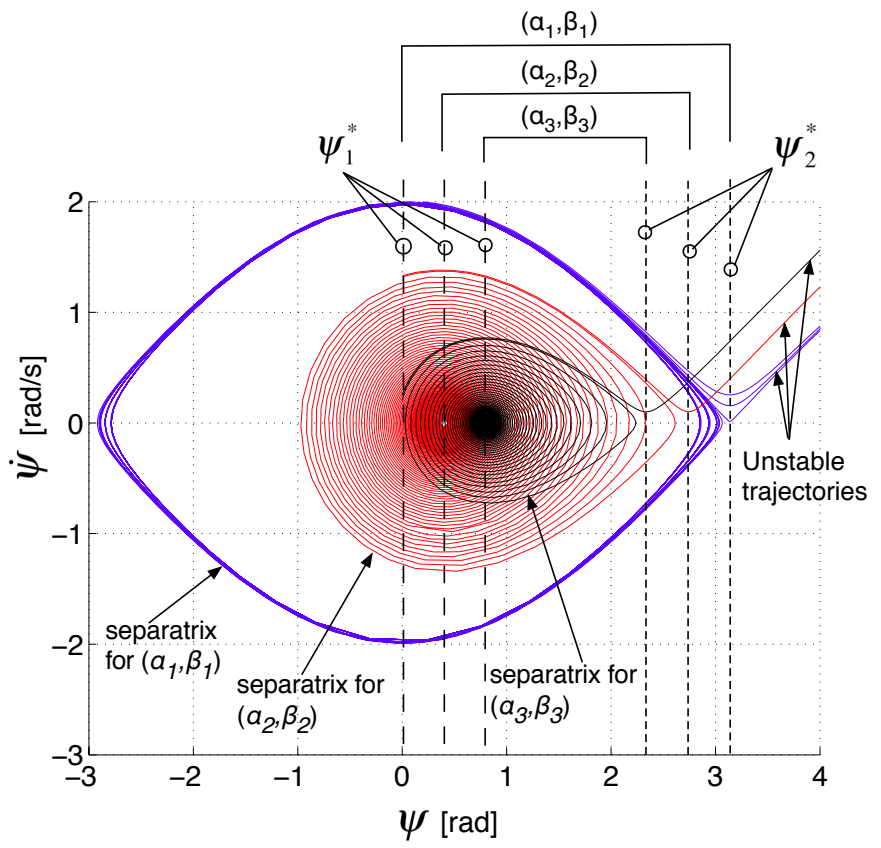

Fig. 7. Phase portrait of slip angle rate, $\dot{\psi}$, versus slip angle $\psi$ for system (25) for three sets of $(\alpha, \beta)$.

the separatrix, bounding the stable state initial conditions in its interior, is labeled. Outside each separatrix, the system is unstable and undergoes complete rotations, i.e. the rotor does not follow the magnetic gradient. Note that increasing the viscous damping shifts the slip angle equilibrium point $\psi_{1}^{*}$, as given by (28), to the right and also reduces the size of the domain of attraction.

For experimental evaluation, it is of interest to predict the maximum angular gradient velocity $\omega$ in (20) that will result in stable rotor rotation when the rotor is started from rest. Assuming that the rotor angle is initialized at the slip angle equilibrium value, this value is given by the maximum slip angle rate achieved on the separatrix. For the undamped case, $\alpha=0$, an analytical expression can be derived using the impulse momentum principle, i.e., by relating the kinetic energy of the system when $\psi=\psi_{1}^{*}$ to the potential energy of the system at $\psi=\psi_{2}^{*}$ through the work done by the load torque,

$$
\begin{aligned}
E\left(\psi_{1}^{*}, \sqrt{\frac{J}{r_{1} F}} \omega_{\max }\right)+W(\beta) & =E\left(\psi_{2}^{*}, 0\right) \\
\frac{1}{2} \frac{J}{r_{1} F} \omega_{\max }^{2}+\int_{\psi_{1}^{*}}^{\psi_{2}^{*}} \beta \mathrm{d} x_{1} & =\int_{\psi_{1}^{*}}^{\psi_{2}^{*}} \sin x_{1} \mathrm{~d} x_{1}(33) \\
\frac{1}{2} \frac{J}{r_{1} F} \omega_{\max }^{2}+\beta\left(\psi_{2}^{*}-\psi_{1}^{*}\right) & =\cos \psi_{1}-\cos \psi_{2} \\
\omega_{\max } & =\sqrt{\frac{r_{1} F}{J}} \sqrt{2\left(\cos \psi_{1}^{*}-\cos \psi_{2}^{*}-\beta\left(\psi_{2}^{*}-\psi_{1}^{*}\right)\right)}
\end{aligned}
$$

where the time scaling factor of (21) has been employed to compute angular velocity. The physical interpretation is that whenever the relative velocity between the rotor and the magnetic gradient exceeds the value $\dot{\psi}=\omega_{\max }$ the rotor becomes unstable and stalls.

2) Constant-velocity Elastic-load Stability: During needle insertions, large forces are typically required to puncture tissue layers, however, the load applied to the needle prior to puncture is not constant, but rather is viscoelastic. As an approximation to the viscoelastic tissue deformation force prior to puncture, this subsection considers the effect of an elastic load on the stability of the actuator during constant velocity operation.

In this case, the MRI gradients are as given in Eq. (20). The actuator rotor synchronizes to the constant gradient frequency and the rack pushes at a constant speed against the elastic material. The load force is given by:

$$
F_{n}=k l \Longrightarrow F_{n}=k R x_{1}
$$

where $k$ is the tissue stiffness constant, and $l$ is the deformation of the elastic load. Equation (26) is rewritten as:

$$
\begin{aligned}
& \dot{x_{1}}=x_{2} \\
& \dot{x_{2}}=-\alpha x_{2}-\sin x_{1}-\gamma x_{1}+\beta
\end{aligned}
$$

where

$$
\begin{aligned}
& \gamma=\frac{k R^{2}}{r_{1} F} \\
& \beta=\frac{b}{\sqrt{J r_{1} F}} \omega+\frac{T_{f}}{r_{1} F}+\frac{k R^{2} \sqrt{J}}{\sqrt{\left(r_{1} F\right)^{3}}} \omega \tau
\end{aligned}
$$




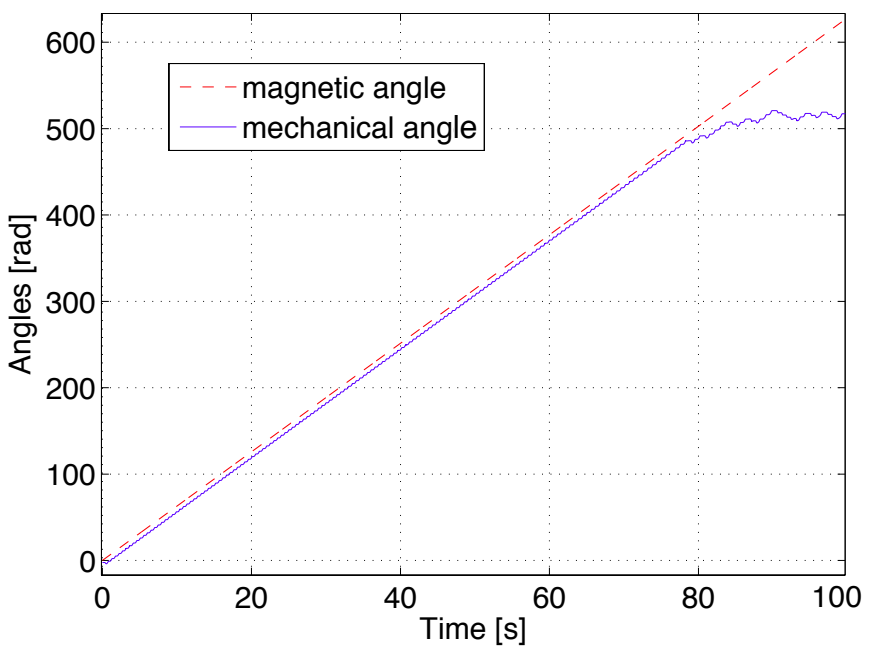

Fig. 8. Simulation of constant-velocity elastic-load response: Rotor angle, $\theta$, and magnetic angle, $\phi$, versus time.

For this case, we can derive a time-dependent equilibrium trajectory for slip angle $\psi^{*}$ as

$$
\psi^{*}=\sin ^{-1}\left(\gamma \omega \tau \sqrt{\frac{J}{r_{1} F}}+\beta+\alpha \omega\right)
$$

and the corresponding matrix $\mathbf{A}$ of the linearized system is given by

$$
A=\left[\begin{array}{cc}
0 & 1 \\
-\cos \psi^{*} & -\alpha
\end{array}\right]
$$

Since the magnetic torque is bounded, it can be anticipated that the system response will be stable for small load torques, but will become unstable as this limit is approached. Simulation was used to examine the transient response and transition to instability of this time-varying system in response to increasing load torque. To obtain qualitative results, the parameter values for Design 1 in Table I were used. In the place of efficiency, the friction parameter values of $T_{f r}=$ $7.0 \times 10^{-5} \mathrm{Nm}$ and $b=1.0 \times 10^{-7} \mathrm{Nms} / \mathrm{rad}$ were used.

Figure 8 shows the evolution of rotor angle and magnetic angle over time, up to the point of instability. Figures 9 and 10 illustrate how the slip angle $\psi$ oscillates about the equilibrium value $\psi_{1}^{*}(t)$. As the force increases, $\psi_{1}^{*}(t) \rightarrow \pi / 2$, the system becomes unstable and the rotor stalls. As can be seen in Fig. 11 , at the point of instability the output force is maximized.

These results indicate that open-loop control of slip angle can be effective in loading scenarios similar to those of needle insertion. This indicates that closed-loop control of needle position can be accomplished with a single control loop that utilizes measurements of needle position (actuator position at the output of the transmission). An MRI-based method for estimating needle position is described in the next section.

\section{MRI-Based Needle Position Sensing \& Closed-LOOP CONTROL}

A variety of MRI-based needle tracking methods are possible. The most straightforward approach for needle interventions is to segment and track the needle in the MR

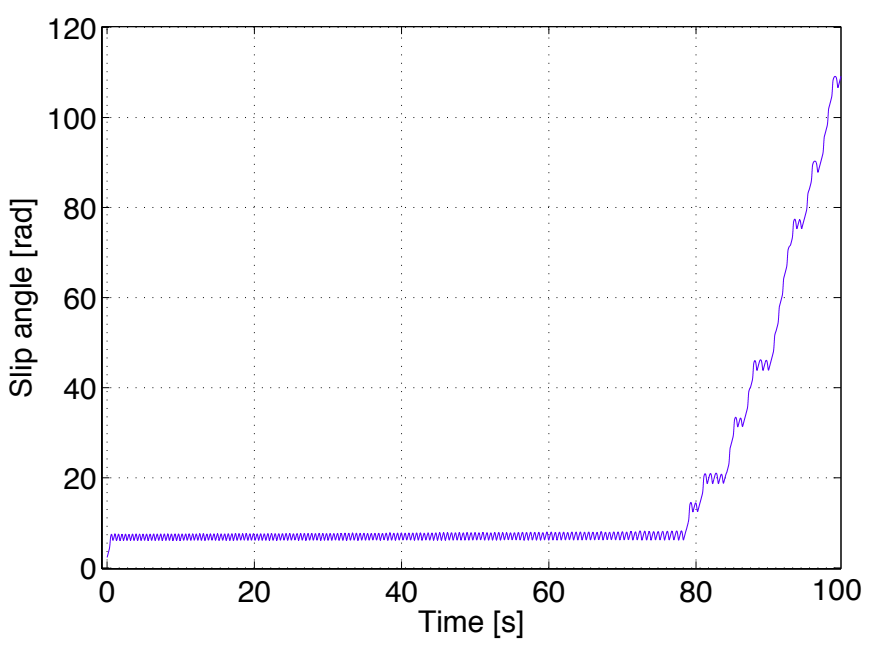

Fig. 9. Simulation of constant-velocity elastic-load response: Slip angle, $\psi$, versus time.

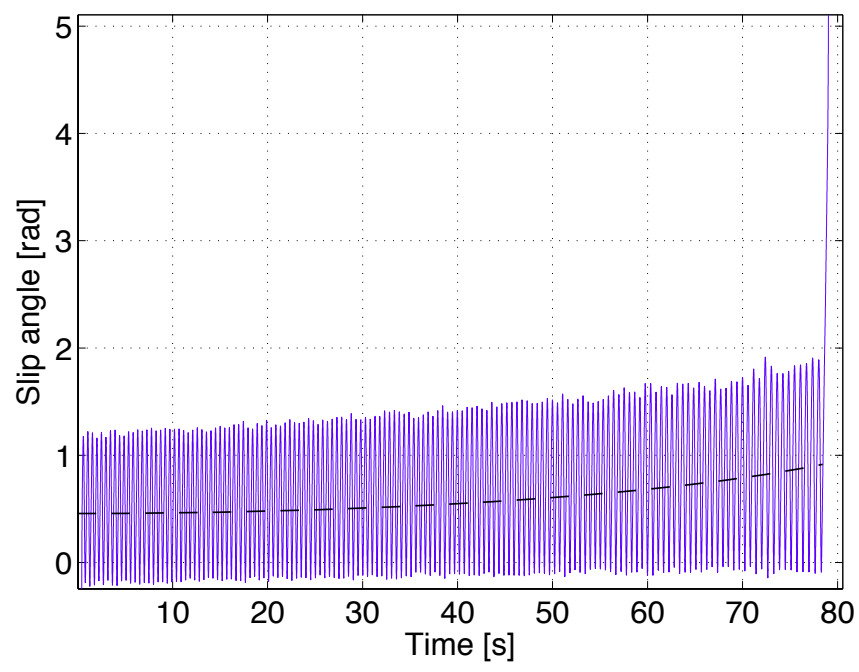

Fig. 10. Expanded scale view of slip angle showing average (dashed) and oscillations leading up to instability.

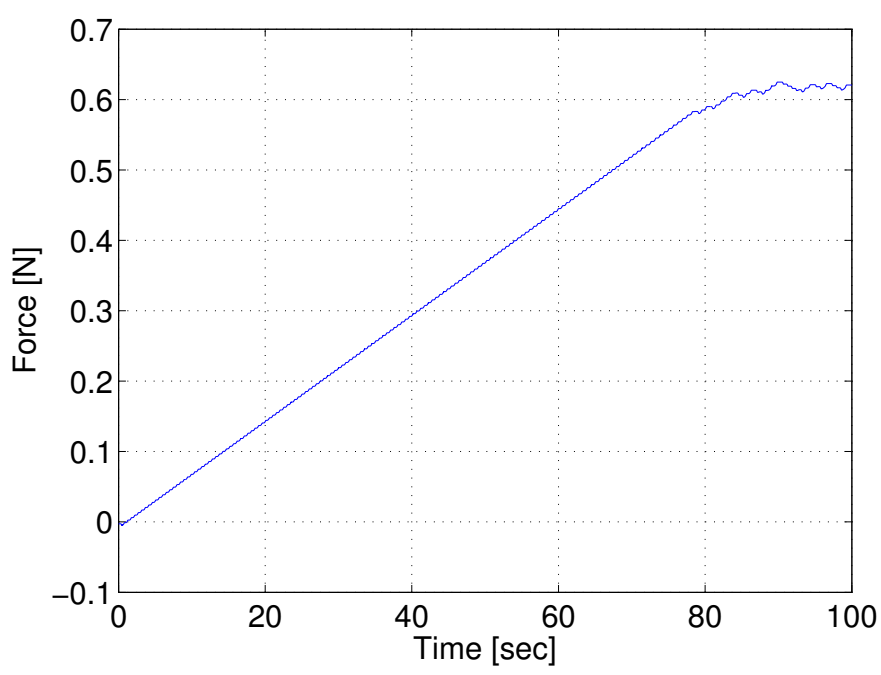

Fig. 11. Output force applied to elastic load versus time. 
image [Lewin, 1999]. Alternatively, fiducial markers can be employed [van der Weide et al., 2001], [Busse et al., 2007] or the propulsive ferromagnetic material itself can be used as a fiducial marker [Felfoul et al., 2008]. A third approach employs miniature receiving coils tuned to the RF frequency of the MRI scanner [Qin et al., 2012]. This approach, however, requires running a cable into the scanner bore and is not considered here since the focus of this paper is on tetherless robots.

There are several challenges to combining tetherless MRIbased actuation and sensing. First, this approach requires the interleaving of imaging and propulsion pulse sequences, which means that propulsion forces are only applied during a portion of each control cycle. Consequently, imaging sequences should be designed to be as fast as possible. Secondly, they should be designed to produce no net propulsive effect. With respect to the first criteria, imaging using standard pulse sequences is unacceptably slow. For example, as reported in [Moche et al., 2008], one of the fastest imaging sequences, TrueFISP, creates an image in $0.4 \mathrm{~s}$.

In the context of millimeter-scale robots propelled through the bloodstream, both criteria have been considered in [Felfoul et al., 2008], [Chanu et al., 2008] where the signature of the ferromagnetic material of the robot produced in the surrounding tissue was used for tracking. Unfortunately, this approach cannot be directly applied to the actuator of Fig. 5 as the ferromagnetic material of the rotor does not generate a magnetic signature since it is not surrounded by tissue or water and consequently is invisible in the MR image.

As an alternative approach, image-based tracking of a fiducial marker attached to the rack, as shown in Fig. 5, is employed. Employing a fiducial marker outside the tissue enables the use of fast single-dimensional projections based on a gradient-echo pulse sequence as exploited in [Chanu et al., 2008] and [Zhang et al., 2010] since the marker is the only MRI-visible object in the $x y$ plane over a range of values along the $x$ axis exterior to the tissue being penetrated.

As described in the next section, this technique resulted in an imaging pulse sequence duration of $12 \mathrm{msec}$. In this sequence, the gradients were applied over only a fraction of this time and were also balanced (see Fig. 12) such that positive and negative pulses were largely offsetting. Furthermore, imaging gradient magnitudes were significantly smaller than actuation magnitudes. Consequently, this approach to MRIbased position sensing provides the speed and non-propulsive properties needed for closed-loop position control.

As shown in Fig. 13, marker position can be estimated from the single-dimensional gradient echo projections using peak detection or correlation. These simple approaches do not work in the presence of nearby tissue, however, since the tissue signal distorts the single-dimensional marker projection. Robust needle tracking can be accomplished, though, through use of a background suppression algorithm [Bergeles et al., 2012]. In this approach, the single-dimensional tissue projection is first recorded prior to the introduction of the fiducial marker into the scanner. During tracking, this background signal, termed $K$, is removed from the recorded single-dimensional

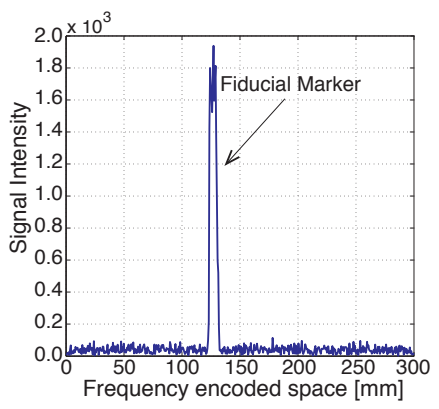

Fig. 13. Fiducial marker position estimation using $x$-axis projections. Plot shows signal intensity versus position in the absence of tissue.

projection, by means of

$$
S^{\prime}=\frac{S}{K+\epsilon}
$$

in which $S$ and $S^{\prime}$ are the original and amplified singledimensional projections, respectively, and $\epsilon=0.1$ is a small constant to avoid division by zero. Direct background subtraction (i.e., $S^{\prime}=|S-K|$ ) is inapplicable because the background signal varies between tracking cycles and $S^{\prime}$ yields false peaks.

\section{A. Closed-loop needle position control}

In needle positioning, it can be problematic to overshoot or oscillate around the goal configuration. Consequently, the controller is designed to advance unidirectionally until the difference between the desired and estimated displacement is less than the control cycle displacement. The control cycle as shown in Fig. 12 consists of a single gradient projection along the $z$ axis for needle tracking. The controller compares the needle position estimate with the desired value and either stops or executes a series of sinusoidal propulsive sequences. The number of propulsive sinusoids applied between tracking sequences can be selected, for example, to correspond to the minimum observable needle displacement.

\section{EXPERIMENTS}

A prototype actuator was constructed to experimentally evaluate the concepts proposed in the preceding sections. Goals included demonstrating the capability to design for clinically relevant needle insertion forces, validating the stability and force production potential of open-loop slip control, and demonstrating closed-loop needle position control using interleaved pulse sequences to perform needle tracking and actuation.

The prototype, shown in Fig. 14, was constructed to achieve an output force of $0.5 \mathrm{~N}$ using the parameters of Design 1 in Table I. It was constructed using $\operatorname{LEGO}^{(R)}$ parts since these plastic components are MRI compatible and enable efficient testing of alternate designs. Given the high component tolerances and friction, however, the transmission efficiency was anticipated to be low in accordance with the design parameter of $\eta=0.2$.

With overall dimensions of $10 \times 10 \times 6 \mathrm{~cm}$, the device is small enough to be positioned at a variety of locations on a 


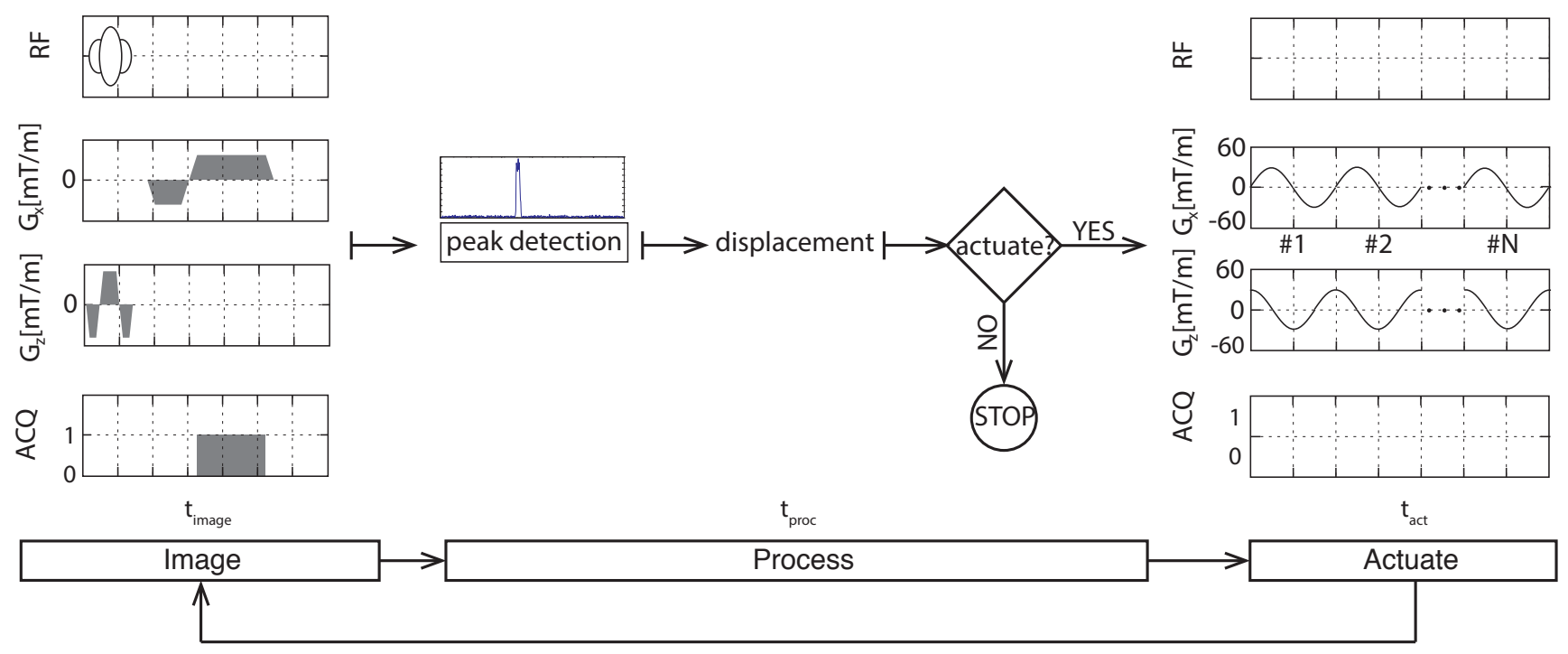

Fig. 12. Controller pulse sequence consisting of interleaved imaging and propulsion pulse sequences.

patient inside the scanner bore. As shown in Fig. 14, a 3mm radius chrome steel ball bearing was safely enclosed in a cavity on the rotor arm. Needle stroke is determined by the length of the rack component, which for the tests performed here was $2.5 \mathrm{~cm}$. The fiducial marker used for needle position estimation is an MR-SPOTS marker (Beekley Medical, CT). For those experiments involving needle insertion, MRI-compatible biopsy needles were used. To evaluate MRI image quality of the assembled system, measurements of the SNR were made by placing the MRI powered actuator next to a porcine heart and it was demonstrated that no SNR deterioration occurred.

Three sets of experiments were conducted. The goal of the first set was to determine if needle insertion under open loop control of slip angle using the prototype actuator was possible. For these experiments, multiple insertion trials were conducted into porcine hearts. The magnetic gradient was rotated at a constant frequency for a sufficient time duration to produce epicardial puncture and subsequent needle insertion. The direction of rotation was then reversed such that the needle was withdrawn from the tissue. These experiments demonstrated successful needle insertion and removal. A video of one insertion appears as multimedia Extension 1. The MRI scanner can be heard in the video soundtrack.

Based on these results, a second set of experiments was performed to provide a detailed evaluation of open loop slip angle control and assessment of maximum force output under conditions of constant input velocity and an elastic needle load as described in Section IV-B2. A third set of experiments was then conducted to evaluate marker-based needle tracking and interleaved imaging and actuation to perform closed-loop needle positioning as described in Section V. All experiments were conducted in a clinical GE 1.5 T MRI scanner (Milwaukee, WI) with pulse sequences programmed using the EPIC API.

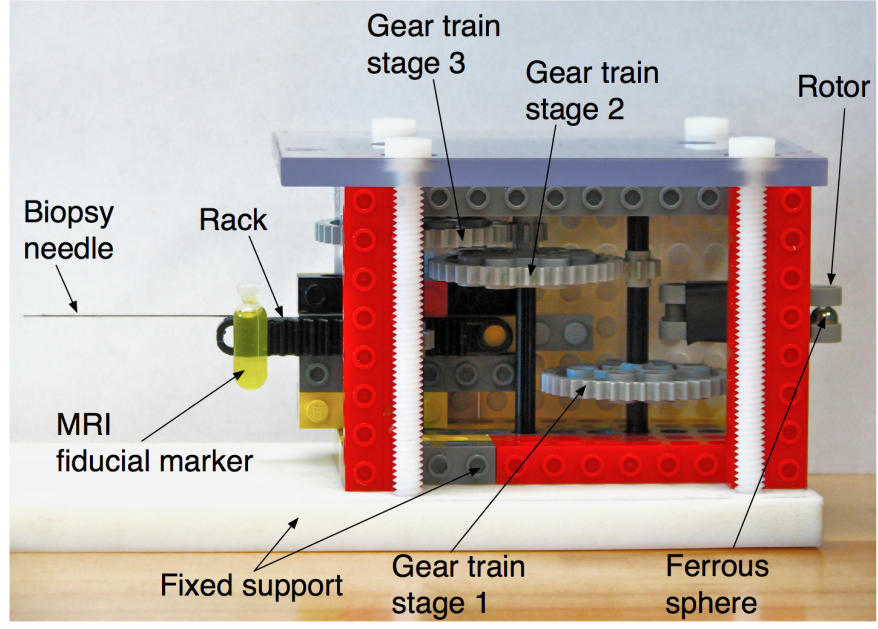

Fig. 14. Actuator prototype with biopsy needle and marker.

\section{A. Constant-Velocity Elastic-Load Experiments}

A series of experiments were conducted to verify the properties of open-loop slip angle control in terms of maximum force output and stability of rotor motion. In these experiments, the magnetic gradient direction was rotated at constant frequency in the horizontal plane causing the rack to stretch an MRIcompatible spring. Rotor angle, gradient angle and output force were recorded as described below.

1) Experimental Method: The experiment is shown in Fig. 15. The mechanical angle was recorded using a digital video camera (Sony HD, 3.1MP, 30fps). The camera was securely mounted $2 \mathrm{~m}$ away from the MRI bore entrance and incorporated into its field of view both the motion of the rotor and the motion of the rack. A mirror was used to reflect the line of sight of the (horizontal) rotor to the (vertical) plane of the image sensor. The mirror was placed at an angle of $45^{\circ}$ so that the reflected motor motion does not exhibit any 
imaging distortion. The mechanical angle was calculated from the reflected image of the rotor using image thresholding, morphological filtering of the resulting binary image to remove noise and speckles, and subsequent selection of the largest connected component. This component corresponded to the rotor, and a line was fit to its contours. The inclination of the line corresponded to the mechanical angle.

The magnetic angle (i.e. the angle of the rotating gradients) was calculated by integrating the constant angular frequency of the rotating magnetic gradients. The elastic load was measured using a calibrated MRI-compatible NiTi spring as shown in Fig. 15. As the rotor rotates, the rack retracts, the spring extends and the load applied to the rack increases.

Two sets of experiments were conducted. In the first set of experiments, magnetic gradient frequency was varied between trials in order to determine the dependence of actuator force and stability on frequency. The second set examined repeatability at a single frequency.

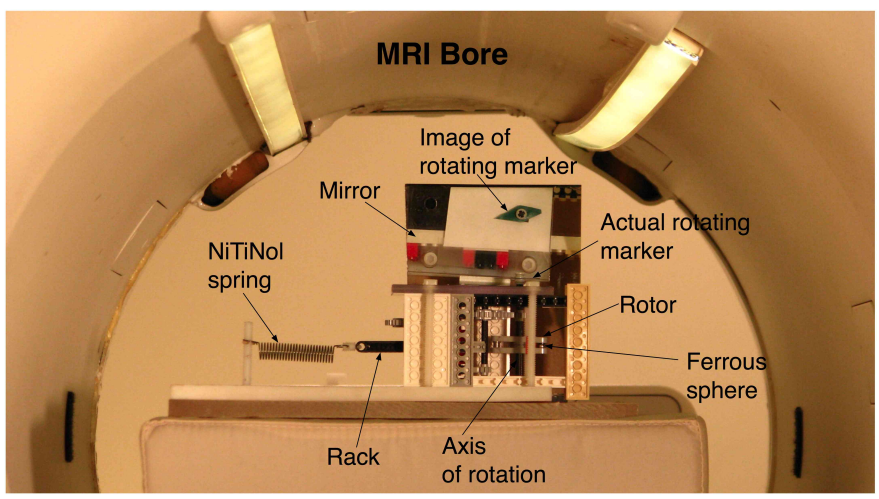

Fig. 15. Experimental design showing actuator at isocenter of MRI bore. Camera located at viewpoint of figure records displacement of calibrated spring and rotor rotation.

2) Results: In the first set of experiments, magnetic gradient frequency was set to values of $f=\{0.4,0.5,0.74,0.83$, $0.91,1.0,1.25,1.43,3\} \mathrm{Hz}$. In each trial, the initial angular speed of the rotor was zero. Figure 16 depicts the maximum output force produced by the actuator at each frequency. The maximum output force is relatively constant up to $1.5 \mathrm{~Hz}$, with a mean value of $520 \mathrm{mN}$ and a standard deviation of $32 \mathrm{mN}$. As shown by the response at $3 \mathrm{~Hz}$, the rotor is unable to synchronize in open loop with the rotating gradient and, consequently, no output force is produced. This effect is predicted in the undamped case by (34), where $\omega_{\max }=14.86$ $\mathrm{rad} / \mathrm{sec}$ or $2.4 \mathrm{~Hz}$ using the parameter values of Design 1 in Table I.

To evaluate the variation in response at a particular frequency that could arise from differences in the configuration of the actuator rotor and gears, eight trials were conducted at a magnetic gradient frequency of $1 \mathrm{~Hz}$. The mean value of the output force was found to be $560 \mathrm{mN}$ with a standard deviation of $27 \mathrm{mN}$. Thus, for all stable rotor frequencies, the actuator was capable of reliably producing the targeted output force magnitude of $0.5 \mathrm{~N}$.

To evaluate the stability of rotor motion during openloop control of slip angle, experimental data from a $1 \mathrm{~Hz}$

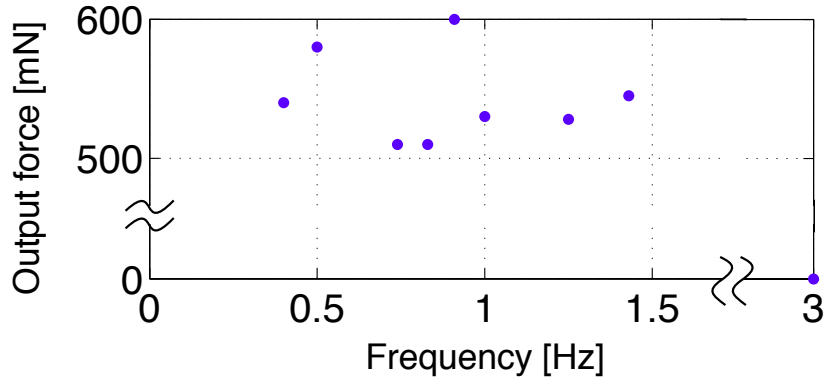

Fig. 16. Maximum spring force as a function of rotor frequency.

trial was compared to the corresponding simulation results in Section IV. The goal is to validate the theoretical model and demonstrate that simulation can be used to reliably predict the actuator response. The mechanical (rotor) and magnetic angles are depicted in Figure 17. Comparing this figure to Figure 8 reveals that, while similar, the experimental response exhibits several short periods at $46 \mathrm{~s}, 63 \mathrm{~s}$ and $65 \mathrm{~s}$ during which the rotor stalls. These are likely due to the use of lowprecision, high-friction components that cause perturbations that temporarily drive the rotor trajectory outside the separatrix for stable rotation.

Figure 18 depicts the slip angle for this experiment and Figure 19 provides a detailed view of slip angle leading up to actuator stall at 46s. Initially, the slip angle oscillates about its slowly increasing equilibrium value, $\psi_{1}$, in a manner comparable to Figures 9 and 10 . At time $t=45 \mathrm{~s}$, however, slip angle temporarily exceeds the maximum stable value of $\pi / 2$ and the rotor stalls for one cycle of the magnetic gradient as shown in Figure 20 before commencing to rotate again. Similar behavior was observed at $62 \mathrm{~s}$ and at to $64 \mathrm{~s}$. From $65 \mathrm{~s}$ to $83 \mathrm{~s}$, mean slip angle increases smoothly until it reaches the critical value of $\pi / 2$ at which point the rotor enters instability and stalls permanently. The maximum output force of $564 \mathrm{mN}$ is produced just before instability as shown in Figure 21. The maximum force predicted by simulation in Section IV was just over $600 \mathrm{mN}$ with the discrepancy likely due to differences in the parameter values used in simulation.

These results demonstrate the capability to perform needle insertion tasks using open-loop slip angle control. They also demonstrate the capability to produce force magnitudes appropriate for clinical needle insertion. Furthermore, it is interesting to note that this actuator design could produce a force of $2.6 \mathrm{~N}$ in the absence of transmission friction $(\eta=1)$. Consequently, output force could easily be substantially increased by optimization of transmission components.

\section{B. Closed-loop Needle Position Control}

As shown above, open-loop control of slip angle can enable needle driving into elastic loads. In this section, closedloop position control is demonstrated using this approach. A fiducial-based method for estimating needle position is first presented. This method is then employed in experiments to drive a needle a specified distance into tissue. 


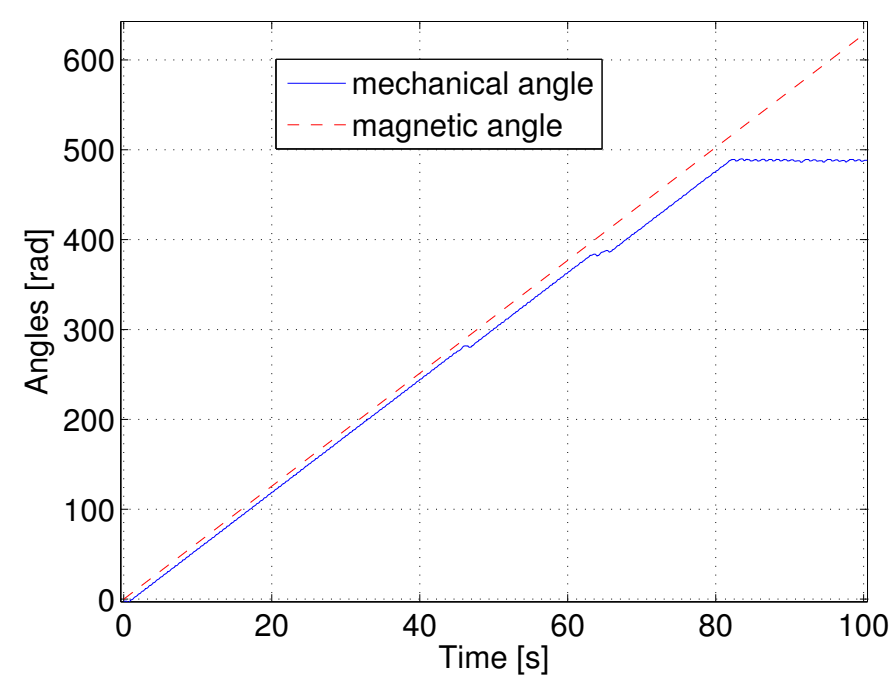

Fig. 17. Mechanical and magnetic angles as functions of time.

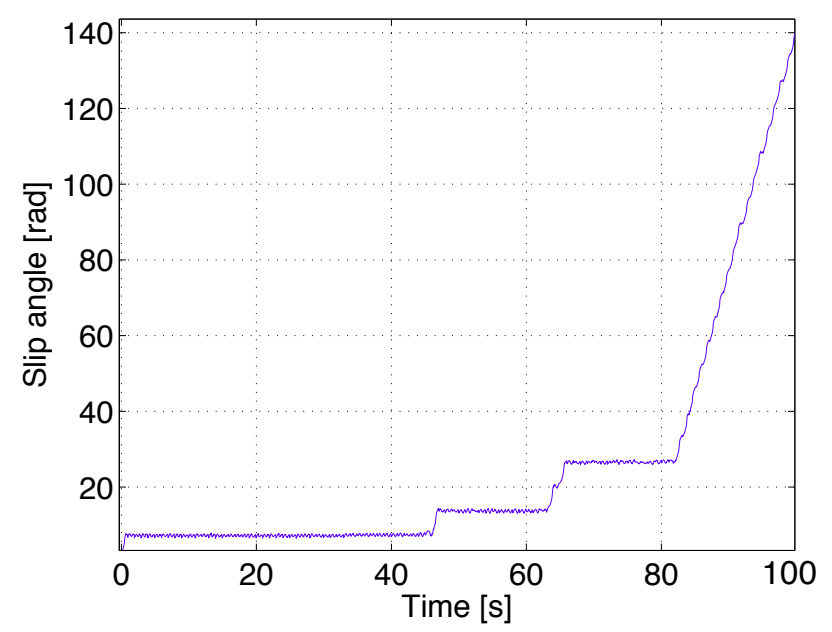

Fig. 18. Slip angle versus time. Note that rotor stalls for one revolution at startup and at times $t=45,62,64 \mathrm{sec}$. Final stall occurs as $t=83 \mathrm{sec}$.

1) Fiducial-based Needle Position Estimation: To evaluate the performance of the localization algorithm of Section V, single-dimensional projections were captured using a gradient echo of $\mathrm{TE}=3 \mathrm{~ms}, \alpha=5^{\circ}$, field-of-view $=300 \mathrm{~mm}$, bandwidth $= \pm 64 \mathrm{KHz}$, matrix $=512$ voxels, resulting in a spatial resolution of $580 \mu \mathrm{m} /$ voxel.

Initially, 10 single-dimensional projections of the tissue (chicken breast) were recorded and an average profile was extracted. Subsequently, a fiducial marker was placed in 5 different locations separated by $20 \mathrm{~mm}$ and the algorithm was used to estimate its location (5 repetitions). Figures 22(a) and (b) show the single-dimensional projection prior to and after background suppression, respectively. To increase the accuracy of peak detection, a gaussian profile was fit to the maximum intensity values of the marker signal and the central value of the fitted gaussian was used [Thörmer et al., 2012]. The average displacement error over all trials was calculated as $-0.65 \pm 1.37 \mathrm{~mm}$, which corresponds to $0-5$ voxels. This is

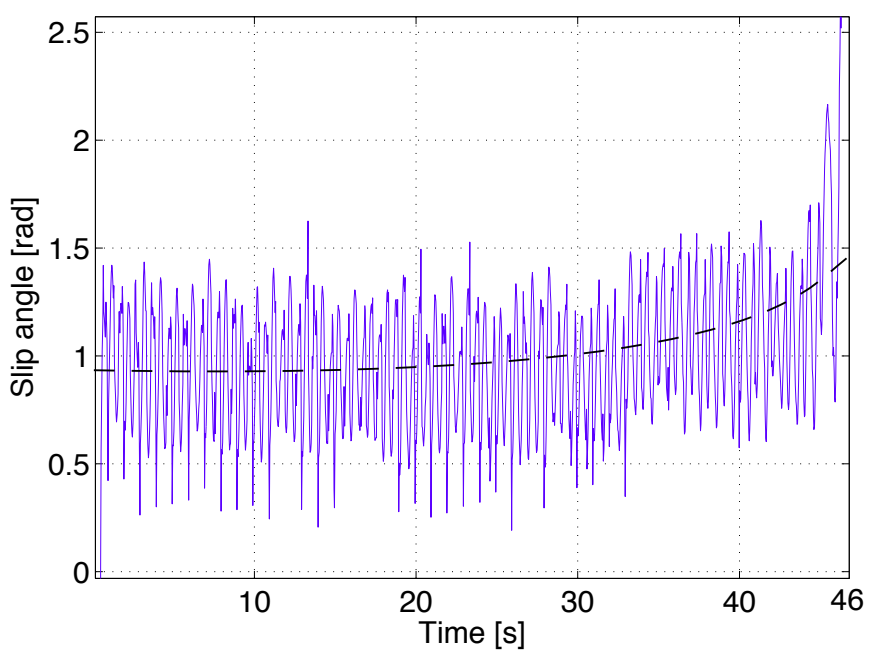

Fig. 19. Slip angle versus time showing increasing mean value up to initial point of stall.

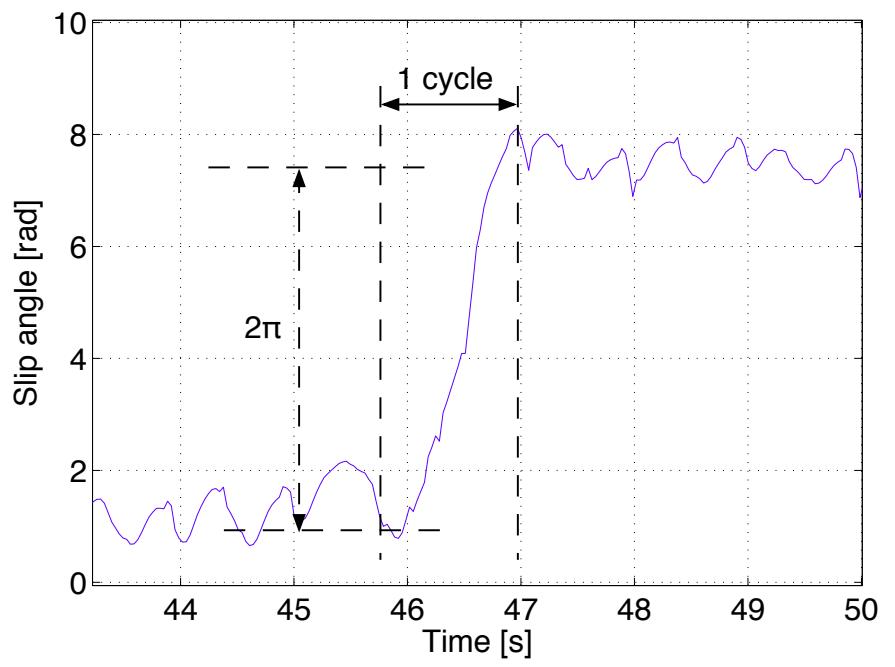

Fig. 20. Slip angle versus time showing rotor stall with mechanical angle falling behind magnetic angle by one revolution $(2 \pi)$.

the expected needle tip localization accuracy and precision for a marker attached to the needle rack.

2) Position Controller: For these experiments, the MRI was connected to an image processing workstation using RTHawk [Santos et al., 2004], resulting in the system network depicted in Figure 23. Needle insertion experiments into chicken breast were conducted with the tissue covered in plastic wrap to avoid dehydration. A 21-gauge bevel-tip MRI-compatible needle was attached to the actuator rack and a small incision was made through the wrap to allow the needle to directly contact the tissue. To provide the background signal that is required for the tracking algorithm, the single-dimensional tissue signal was captured in the absence of the MRI-powered actuator and the fiducial marker. Subsequently, the actuator was positioned in proximity to the tissue, the needle was aligned with the incision in the plastic wrap, and an initial image volume, as shown in Figure 24(a), was captured using 3-plane gradient echoes with $\mathrm{TE}=2.4 \mathrm{~ms}, \mathrm{TR}=6.7 \mathrm{~ms}$, bandwidth $= \pm 31.3 \mathrm{KHz}$, 


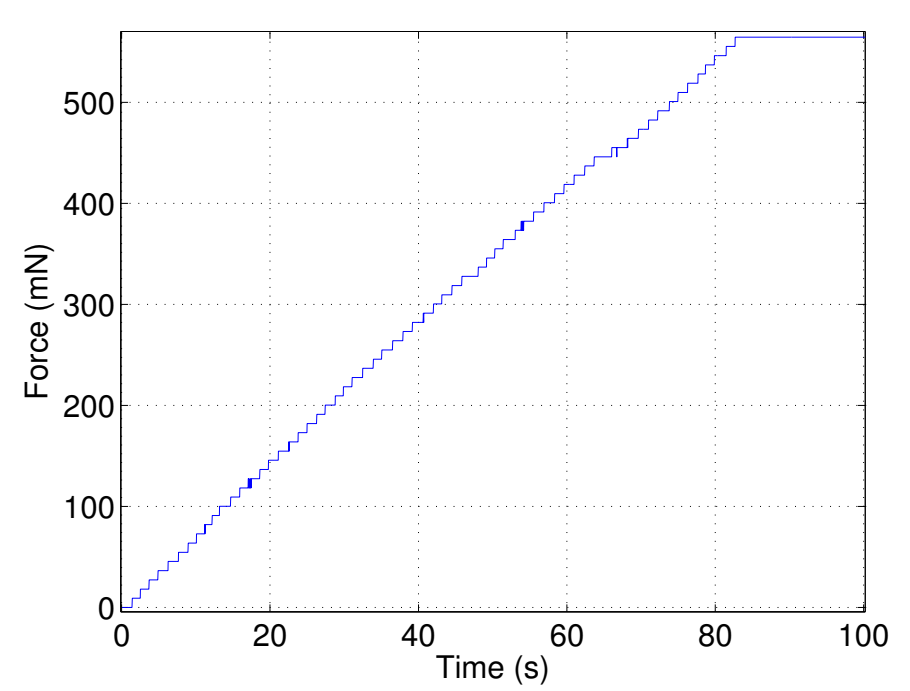

Fig. 21. Spring force versus time.

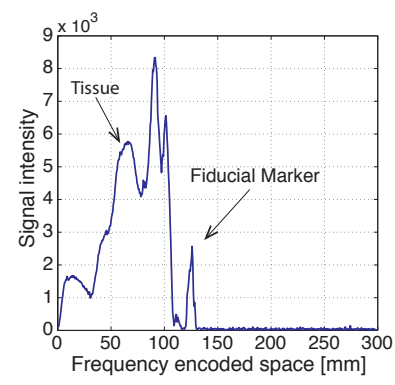

(a)

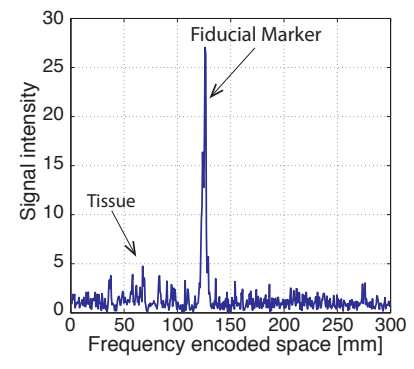

(b)
Fig. 22. Fiducial marker signal amplification. (a) Signal intensity versus position in the presence of tissue. (b) Amplified signal intensity versus position obtained applying (42) to (a).

$\alpha=30^{\circ}$, fov $=300 \mathrm{~mm}$.

In these experiments, the goal is to advance the tip of the needle by a specified displacement. Under the reasonable assumption that the needle is longitudinally rigid, this is equivalent to translating the fiducial marker by the same distance. Given that each rotation of the rotor corresponds to a needle displacement of $250 \mu \mathrm{m}$, the controller pulse sequence is selected to consist of $N=5$ propulsion cycles (see Figure 12) corresponding to $1.25 \mathrm{~mm}$ of needle displacement per imaging pulse sequence. This displacement is selected to be the largest integer number of cycles that does not exceed the standard deviation of estimator displacement error, $1.37 \mathrm{~mm}$, as measured by the fiducial-based estimator.

The control cycle timing, as labeled in Figure 12, consists of an imaging time, $t_{\text {image }}=12 \mathrm{msec}$, a computational and data transfer time of $t_{\text {proc }}=200 \mathrm{msec}$ and an actuation time of $t_{\mathrm{act}}=5 \mathrm{sec}$ (rotor rotation rate of $1 \mathrm{~Hz}$ ).

For the trial depicted in Figure 24, the desired displacement was selected as $10 \mathrm{~mm}$. Following the control cycle of Figure 12 and the block diagram of Figure 23, fiducial marker position was first estimated using a tracking pulse sequence and compared to the desired final position. A propulsion sequence was then performed as long as the remaining distance exceeded the propulsion displacement. Final estimated needle

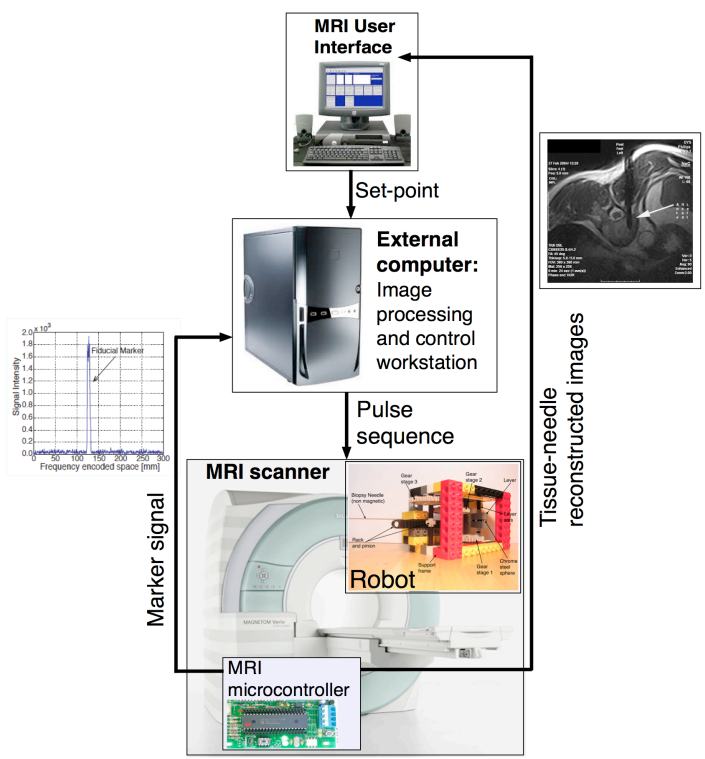

Fig. 23. System network architecture for closed loop control.

displacement for this trial was $9.92 \mathrm{~mm}$.

While eight actuation cycles correspond to exactly $10 \mathrm{~mm}$, it was observed that the controller ran for 12 cycles. This indicates that, similar to Figure 18, open-loop control of slip angle resulted in four cycles in which the rotor stalled. A final 3-plane gradient echo image was captured to evaluate and verify the displacement of the needle and fiducial marker and is shown in Figure 24(b). Needle displacement was confirmed to be $10 \mathrm{~mm}$ ( $1 \mathrm{~mm}$ screen resolution) using the scanner console interface by manually selecting the marker centroid before and after displacement. The absence of artifacts in Figure 24 also demonstrates the MR-compatibility of the actuator technology.

\section{CONCLUSION}

The MRI-powered actuator technology proposed and demonstrated in this paper offers the potential of enabling lowcost MRI-guided robotic interventions. Since the actuators can be constructed entirely from plastic and ceramic components encasing one or more standard ball bearings, the overall design is simpler and much less inexpensive than existing MR-compatible robotic actuator technologies. Furthermore, no wires or transmission lines need be run into the scanner bore and, by design, actuator sensing and control is completely integrated with the MR imaging system such that no peripheral devices are required.

Conditions for MRI compatibility are satisfied since all materials except for the ball bearings are nonmagnetic and nonconductive. The ball bearings are safely encased in the actuator to prevent their escape and are small enough in volume that they can be located at sufficient distance from the tissue of interest so as to not affect imaging SNR.

The paper provides the fundamental components needed to implement, as well as to further develop, the technology. These include design guidelines to produce application-specific force 


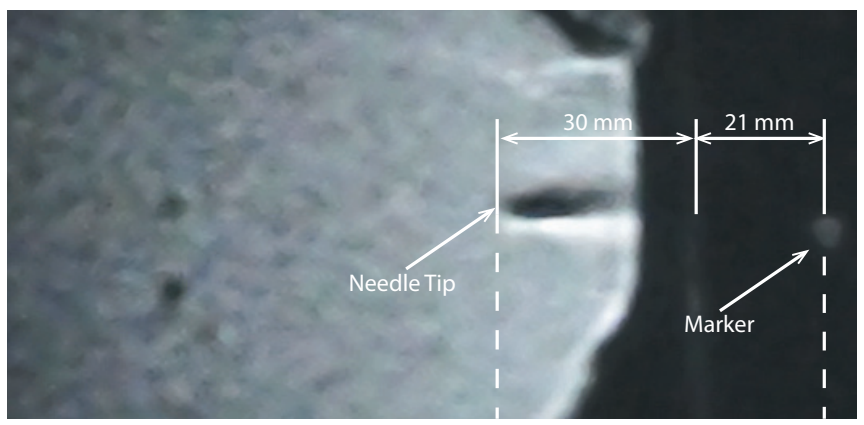

(a)

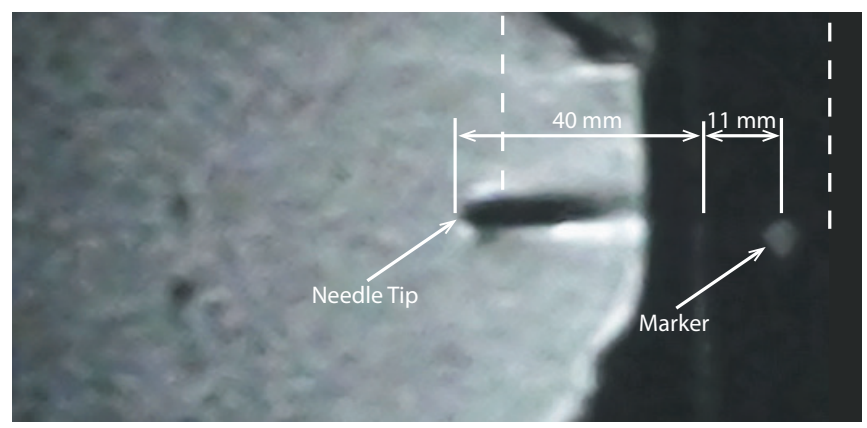

(b)

Fig. 24. MRI images of position-controlled $10 \mathrm{~mm}$ needle displacement into chicken breast. (a) Image prior to displacement showing needle tip location and fiducial marker. (b) Image at completion of motion.

levels as well as techniques for closed-loop MRI-based actuator state estimation and control. These methods were validated by constructing and testing an actuator designed to produce a maximum force sufficient for porcine spleen insertion. In addition, design specifications appropriate for human prostate needle insertion are also detailed.

There are a number of opportunities to improve this technology through additional research. For example, while openloop control of slip angle was demonstrated analytically and experimentally to be adequate for needle insertion, there may be applications for which direct control of rotor angle is necessary. Implementing commutation control introduces new challenges and initial work on this topic is reported in [Bergeles et al., 2013].

A second topic of interest is to develop actuator designs that incorporate bearings and transmission elements that are both low friction and low cost. If these goals can be met simultaneously, it may be possible to develop single-use actuators for MRI-guided procedures.

As a third topic for future research, many interventional applications require actuating multiple degrees of freedom. A variety of approaches to this problem are currently being investigated. First, full advantage can be taken of the three independent coordinate-direction gradient inputs. In addition, multi-actuator control can be achieved through the use of locking mechanisms as described in [Vartholomeos et al., 2011] as well as through the techniques currently being developed for the independent control of magnetic milli- and micro-robots [Vartholomeos et al., 2012].

\section{APPENDIX}

InDEX TO Multimedia EXTENSIONS

The multimedia extensions to this article are at: http://www.ijrr.org/.

\begin{tabular}{cll} 
Extension & Type & Description \\
\hline 1 & Video & MRI-powered needle insertion into a porcine heart. \\
\hline
\end{tabular}

\section{REFERENCES}

[Abbott et al., 2007] Abbott, J. J., Ergeneman, O., Kummer, M. P., Hirt, A. M., and Nelson, B. J. (2007). Modeling magnetic torque and force for controlled manipulation of soft-magnetic bodies. IEEE Trans. Robotics, 23(6):1247-1252.

[Arcese et al., 2011] Arcese, L., Fruchard, M., Beyeler, F., Ferreira, A., and Nelson, B. J. (2011). Adaptive backstepping and MEMS force sensor for an mri-guided microrobot in the vasculature. IEEE Int. Conf. Robotics and Automation, pages 4121-4126.

[Barth, 1973] Barth, H. V. (1973). Ultrasonic driven motor. IBM Technical disclosure bulletin, (2236).

[Bergeles et al., 2012] Bergeles, C., Qin, L., Vartholomeos, P., and Dupont, P. E. (2012). Tracking and position control of an MRI-powered needleinsertion robot. IEEE Int. Conf. Engineering in Medicine and Biology, pages 928-931.

[Bergeles et al., 2013] Bergeles, C., Vartholomeos, P., Qin, L., and Dupont, P. E. (2013). Closed-loop commutation control of an MRI-powered robot actuator. IEEE Int. Conf. Robotics and Automation, pages 690-695.

[Busse et al., 2007] Busse, H., Trampel, R., Gruünder, W., Moche, M., and Kahn, T. (2007). Method for automatic localization of MR-visible markers using morphological image processing and conventional pulse sequences: feasibility for image-guided procedures. J. Magnetic Resonance Imaging, 28:1087-1096.

[Chanu et al., 2008] Chanu, A., Felfoul, O., Beaudoin, G., and Martel, S. (2008). Adapting the clinical MRI software environment for real-time navigation of an endovascular untethered ferromagnetic bead for future endovascular interventions. Magnetic Resonance in Medicine, 59:12871297.

[Chinzei et al., 2000] Chinzei, K., Hata, N., Jolesz, F. A., and Kikinis, R. (2000). MR compatible surgical assist robot: system integration and preliminary feasibility study. Int. Conf. Medical Image Computing and Computer Assisted Intervention, pages 921-930.

[Felfoul et al., 2008] Felfoul, O., Mathieu, J.-B., Beaudoin, G., and Martel, S. (2008). In vivo MR-tracking based on magnetic signature selective excitation. IEEE Trans. Medical Imaging, 27(1):28-35.

[Fischer et al., 2008] Fischer, G. S., Krieger, A., Iordachita, I., Csoma, C., Whitcomb, L. L., and Fichtinger, G. (2008). MRI compatibility of robot actuation techniques - a comparative study. Int. Conf. Medical Image Computing and Computer Assisted Intervention, pages 509-517.

[Giffin et al., 2010] Giffin, A., Shneider, M., Chiranjeev, K. S., Ames, T. L., and Miles, R. B. (2010). Effects of a conducting sphere moving through a gradient magnetic field.s. AIAA Aerospace Sciences Meering, Orlando, Florida.

[Han et al., 2008] Han, B. H., Park, S., and Lee, S. Y. (2008). Gradient waveform synthesis for magnetic propulsion using mri gradient coils. Physics in Medicine and Biology, 53:4639.

[Lewin, 1999] Lewin, J. S. (1999). Interventional MR imaging: concepts, systems, and applications in neuroradiology. Am. J. Neuroradiology, 20:735-748.

[Li et al., 2011] Li, M., Kapoor, A., Mazilu, D., and Horvath, K. A. (2011). Pneumatic actuated robotic assistant system for aortic valve replacement under MRI guidance. IEEE Trans. Biomedical Engineering, 58(2):443451.

[Martel et al., 2009] Martel, S., Felfoul, O., Mathieu, J., Chanu, A., Tamaz, S., Mohammadi, M., Mankiewicz, M., and Tabatabaei, N. (2009). MRIbased medical nanorobotic platform for the control of magnetic nanoparticles and flagellated bacteria for target interventions in human capillaries. Int. J. Robotics Research, 28(9):1169-1182.

[Martel et al., 2007] Martel, S., Mathieu, J.-B., Felfoul, O., Chanu, A., Aboussouan, E., Tamaz, S., Pouponneau, P., Yahia, L., Beaudoin, G., Soulez, G., and Mankiewicz, M. (2007). Automatic navigation of an untethered device in the artery of a living animal using a conventional clinical magnetic resonance imaging system. Applied Physics Letters, 90:114105 
[Masamune et al., 1995] Masamune, K., Kobayashi, E., Masutani, Y., Suzuki, M., Dohi, T., and et al (1995). Development of an mri-compatible needle insertion manipulator for stereotactic neurosurgery. J. Image Guided Surgery, 1:242-248.

[Maurin et al., 2004] Maurin, B., Barbe, L., Bayle, B., Zanne, P., Gangloff, J., de Mathelin, M., Gangi, A., Soler, L., and Forgione, A. (2004). In vivo study of forces during needle insertions. Perspective in Image-Guided Surgery: $M R N V$, pages 415-422.

[McNab et al., 2012] McNab, J. A., Witzel, T., Bhat, H., Heberlein, K., Keil, B., Cohen-Adad, J., Tisdall, M., and Wald, L. (2012). In vivo human brain measurements of axon diameter using $300 \mathrm{mt} / \mathrm{m}$ maximum gradient strengths. Int. Symp. Magnetic Resonance in Medicine, (20).

[Moche et al., 2008] Moche, M., Trampel, R., Kahn, T., and Busse, H. (2008). Navigation concepts for MR image-guided interventions. $J$. Magnetic Resonance Imaging, 27:276-291.

[Patriciu et al., 2007] Patriciu, A., Petrisor, D., Muntener, M., Mazilu, D. Schar, M., and Stoianovici, D. (2007). Automatic brachytherapy seed placement under mri guidance. IEEE Trans. Biomedical Engineering, 54:1499-1506.

[Podder et al., 2006] Podder, T., Clark, D., Sherman, J., Fuller, D., Messing, E., Rubens, D., Strang, J., Brasacchio, R., Liao, L., Ng, W.-S., and Yu, Y. (2006). In vivo motion and force measurement of surgical needle intervention during prostate brachytherapy. Medical Physics, 33:2915.

[Qin et al., 2012] Qin, L., Schmidt, E. J., Tse, Z. T. H., Santos, J. M., Hoge, W. S., Tempany-Afdhal, C., Butts-Pauly, K., and Dumoulin, C. L. (2012). Prospective motion correction using tracking coils. Magnetic Resonance in Medicine.

[Santos et al., 2004] Santos, J. M., Wright, G. A., and Pauly, J. M. (2004). Flexible real-time magnetic resonance imaging framework. IEEE Int. Conf. Engineering in Medicine and Biology, pages 1048-1051.

[Song et al., 2011] Song, D. Y., Burdette, E. C., Fienne, J., Armour, E., Kronreif, G., Deguet, A., Zhang, Z., Iordachita, I., Fichtinger, G., and Kazanzides, P. (2011). Robotic needle guide for prostate brachytherapy: clinical testing of feasibility and performance. Brachytherapy, 10:57-63.

[Su et al., 2012] Su, H., Cardona, D. C., Shang, W., Camilo, A., Cole, G. A., Rucker, D. C., Webster III, R. J., and Fischer, G. S. (2012). A MRI-guided concentric tube continuum robot with piezoelectric actuation: a feasibility study. IEEE Int. Conf. Robotics and Automation, pages 1939-1945.

[Thörmer et al., 2012] Thörmer, G., Garnov, N., Moche, M., Haase, J., Kahn, T., and Busse, H. (2012). Simultaneous 3D localization of multiple MRvisible markers in fully reconstructed MR images: proof-of-concept for subsecond position tracking. Magnetic Resonance Imaging, 30:371-381.

[Tokuda et al., 2012] Tokuda, J., Song, S.-E., Fischer, G. S., Iordachita, I., Seifabadi, R., Cho, B. J., and Fichtinger, G. (2012). Preclinical evaluation of MRI-compatible pneumatic robot for angulated needle placement in prostate interventions. Int. J. Computer Assisted Radiology and Surgery, pages $1-9$.

[Tsekos et al., 2005] Tsekos, N., Ozcan, A., and Christoforou, E. (2005). A prototype manipulator for mr-guided interventions inside standard cylindrical mri scanner. J. Biomechanical Engineering, (127):972 -980.

[Tsekos et al., 2007] Tsekos, N. V., Khanicheh, A., Christoforou, E., and Mavroidis, C. (2007). Magnetic resonance compatible robotic and mechatronic systems for image-guided interventions and rehabilitation: a review study. Annual Review of Biomedical Engineering, 9:351-387.

[van der Weide et al., 2001] van der Weide, R., Bakker, C. J. G., and Viergever, M. A. (2001). Localization of intravascular devices with paramagnetic markers in MR images. IEEE Trans. Biomedical Engineering, 20(10):1061-1071.

[Vartholomeos et al., 2012] Vartholomeos, P., Akhavan-Sharif, M. R., and Dupont, P. E. (2012). Motion planning for multiple millimeter-scale magnetic capsules in a fluid environment. IEEE Int. Conf. Robotics and Automation, pages 1927-1932.

[Vartholomeos et al., 2011] Vartholomeos, P., Qin, L., and Dupont, P. E. (2011). MRI-powered actuators for robotic interventions. IEEE/RSJ Int. Conf. Intelligent Robots and Systems, pages 4508-4515.

[Washio and Chinzei, 2004] Washio, T. and Chinzei, K. (2004). Needle force sensor, robust and sensitive detection of the instant of needle puncture. Int. Conf. Medical Image Computing and Computer Assisted Intervention, pages 113-120.

[Zhang et al., 2010] Zhang, K., Krafft, A. J., Umathum, R., Maier, F., Semmler, W., and Bock, M. (2010). Real-time MR navigation and localization of an intravascular catheter with ferromagnetic components. Magnetic Resonance Materials in Physics, Biology and Medicine, 23(3):153-163. 